\title{
Hydrogen Abstraction/Addition Reactions in Soot Surface Growth
}

\author{
Qingzhao Chu ${ }^{1,2}$, Baolu Shi ${ }^{1}$, Hongyu Wang ${ }^{2}$, Dongping Chen ${ }^{2 *}$, Lijuan Liao ${ }^{3 *}$ \\ ${ }^{1}$ School of Aerospace Engineering, Beijing Institute of Technology, Beijing, 100081, China \\ ${ }^{2}$ State Key Lab of Explosion Science and Technology, Beijing Institute of Technology, Beijing, 100081, \\ China \\ ${ }^{3}$ Key Laboratory for Mechanics in Fluid Solid Coupling Systems, Institute of Mechanics, Chinese \\ Academy of Sciences, Beijing 100190, China
}

\begin{abstract}
The hydrogen abstraction (HB) and addition reactions (HD) by H radicals are examined on a series of polycyclic aromatic hydrocarbon (PAH) monomers and models of quasi-surfaces using quasi-classical trajectory (QCT) method. The QCT results reproduce the rate constants of HB reactions on PAH monomers from density function theory (DFT) in the range of 1500-2700 K. The PAH size has a minor impact on the rates of $\mathrm{HB}$ reactions especially at temperatures beyond $2100 \mathrm{~K}$. By contrast, HD reactions have a clear size dependence and a larger PAH yields a higher rate. It is also found that the preferred reaction pathway changing from $\mathrm{HB}$ to $\mathrm{HD}$ reactions at $\sim 1900 \mathrm{~K}$. The rates of surface $\mathrm{HB}$ and $\mathrm{HD}$ reactions exceed those in the gas phase by nearly a factor of magnitude. Further analysis on the detailed trajectory of QCT method reveals that about $50 \%$ of the surface reactions can be attributed to the events of surface diffusion, which depends on the local energy transfer in the gas-surface interactions. However, this phenomenon is not preferred in PAH monomers as expected. Our finding here highlights the misinterpretation of surface reactions as the product of the first collision between gaseous species and particle surface, and surface diffusion induced reactions should be accounted for in the rates of surface $\mathrm{HB}$ and HD reactions. Rate constants of $\mathrm{HB}$ and HD reactions on each reactive site (surface zig-zag, surface free-edge and pocket free-edge sites) are calculated by QCT method, which are recommended for the further development of surface chemistry models in soot formation.
\end{abstract}

Keywords: Surface reaction; HACA; Collision theory; Molecular dynamics; Quasi-classic trajectory

\footnotetext{
*Corresponding author. Tel: +8613167557765. E-mail: dc516@bit.edu.cn (Dongping Chen)

Tel: +86-10-8254-4291. E-mail: liaohuanxin@hotmail.com (Lijuan Liao)
} 


\section{Introduction}

Soot particles have attracted intensive public attention due to their hazardous effects on environment and human health [1]. Soot formation involves the chemistry of precursors, particle nucleation and mass/size growth [2]. The surface reactions of soot play an important role in its mass growth, involving interactions between gaseous species and active sites on particle surface. Thus, the influence of surface structure on its reactivity is critical to infer the mass growth of soot.

Polycyclic aromatic hydrocarbons (PAHs) are accepted as the precursors of soot and also its main building block $[3,4]$. Many experimental investigations and chemical kinetic modelings have been performed on PAHs to understand the mechanism in soot surface reactions $[5,6]$. Since the calculation of reactions involving large aromatic molecules is quite time-consuming, it is well established to infer the reaction pathways and rate constants of large hydrocarbons using smaller species via the rate-rule assumption. Transition state theory (TST) is one of the most common methods for estimating the rate constants. A series of studies using the TST approach have been conducted to examine the effect of PAH size on the reactivity. Hou et al. [7] computed the energetics of hydrogen abstraction from benzene and naphthalene using the method of density functional theory (DFT), and they found that M06-2X/6$311 \mathrm{G}(\mathrm{d}, \mathrm{p})$ has the best performance with errors within $1 \mathrm{kcal} / \mathrm{mol}$ compared to the $\mathrm{CCSD}(\mathrm{T}) / \mathrm{CBS}$ calculations. It was also revealed that the effects of PAH sizes, structures, and reaction sites on the rate constants of hydrogen abstraction reactions are limited from benzene to pentacene due to the similar energy barriers. Liu et al. [8] have reported that the hydrogen-abstraction/acetylene-addition (HACA) reactions are sensitive to the site type. According to the local structure, reactive sites are divided into three types, i.e., free-edge, zig-zag and armchair site. Among all three site types, free-edge site is the most reactive one. For hydrogen abstraction reactions, it was found that the difference in the energy barrier over all site types is less than $1 \mathrm{kcal} / \mathrm{mol}$, and the obvious deviation (e.g. $41 \%)$ in rate constants is attributed 
to the effect of orientation hindrance, where the reactive site is hindered by local geometry. As discussed above, the TST method can well predict the rate constants for PAH molecules up to pentacene, but this method becomes too expensive for large PAHs and soot particles.

The surface HACA model developed by Frenklach and coworkers [9] has been widely applied to address the mass growth due to surface reactions. In this model, the rate of surface reaction $\left(R_{\mathrm{s}}\right)$ is determined by $R_{s}=\alpha \chi_{i} A_{s} R_{g}$, where $\alpha$ is an empirical parameter accounting for the probability of a gaseous molecule colliding with reactive sites on particle surface, $\chi_{i}$ is the number density of reactive site $i, A_{s}$ is the particle surface area and $R_{\mathrm{g}}$ is the reaction rate of an analogous gas-phase reaction. The $R_{\mathrm{g}}$ was adapted from the hydrogen abstraction on benzene according to the rate-rule assumption, and the activation energy barrier was lowered by $3 \mathrm{kcal} / \mathrm{mol}$ based on the assumption that the reactive $\mathrm{C}-\mathrm{H}$ sites locate at the aromatic bay [10]. This approximation is somewhat arbitrary and might not be applicable for all situations. Therefore, an alternative method is required to examine the site effect and quantify the surface reaction rates. However, the available experimental methods are not able to extract the surface reactivity of soot at the atomic level. Recently, molecular dynamics (MD) simulations have become popular in the research of soot formation, for instance, soot particles inception $[11,12]$ and the morphology evolution [13] of soot particles. Chen et al. [14] have successfully built microscopic structures of PAH clusters to mimic soot particles, and managed to estimate surface properties including surface area, number density of reactive sites and parameter $\alpha$. They also found that surface shallow regions (e.g. pockets) are frequently formed owing to the surface rearrangement of PAH molecules [15]. It was speculated that the reactivity of reactive site in the pocket region is different from its counterpart, however, this has not been further investigated in detail.

Besides TST and MD methods, quasi-classical trajectory (QCT) is an alternative method using classical mechanics to describe the collision between a target molecule and a collision partner [16], which 
is widely applied to extract the rate constants of bimolecular reactions in the gas phase [17-19]. Compared to TST method, QCT method examines the detailed characteristics of collision and energy transfer with a higher computing cost. Fu et al. [20] reported a high-level computational study of roaming dynamics in the bimolecular combustion reaction $\mathrm{H}+\mathrm{C}_{2} \mathrm{H}_{4}=\mathrm{H}_{2}+\mathrm{C}_{2} \mathrm{H}_{3}$. It was learnt that the binary collision can lead to "collision-induced" roaming, i.e., the incident $\mathrm{H}$ radical roams over the $\mathrm{C}_{2} \mathrm{H}_{4}$ molecule before a hydrogen abstraction reaction occurs. This unique behavior is an important contributor in the overall reaction rate, but it cannot be accessed by any conventional minimum-energy path (e.g. TST approach). In the study of soot surface reactions, no similar work is available, and this motivates us to explore the collision dynamics of surface hydrogen abstraction and addition reactions using QCT method.

In this paper, the potential collisions between the incident species and soot surface were explored using QCT method to reveal the detailed dynamics of surface reactions in soot formation. Firstly, molecular structures are analyzed to obtain solvent-excluded surface (SES) area and percentages of surface hydrogen. The detailed collision dynamics are then extracted from the quasi-classical trajectories to analyze the collision cross sections and reaction rate constants. Furthermore, the local properties of each site, including per-site SES area, available incident angle, local van der Waals potential energy surface, are discussed to illustrate the site effects on collision dynamics. The detailed dynamics of surface reactions are examined to identify the impact of gas-surface scattering. Finally, the rate constants of hydrogen abstraction and addition reactions on each site are recommended for soot surface models.

\section{Computational methods}

\subsection{Quasi-classical trajectory}

Two representative models are selected in this work to reveal the collision dynamics of hydrogen atoms with polycyclic aromatic hydrocarbons. We firstly consider PAH monomers of benzene (A1), coronene (A7) and circumcoronene (A19) to mimic the interactions between $\mathrm{H}$ atoms in gaseous reactions. 
Two quasi-surface configurations are further constructed using both A7 and A19, termed as A7s and A19s. The selection of PAH size is based on the recent studies of mass spectra [21] and high-resolution atomic force microscopy [22] in sooting flames, indicating that soot particles are composed of PAHs with a mass ranging from 100 to $700 \mathrm{amu}$

QCT calculations were performed using the above two models to compare the dynamics of hydrogen abstraction/addition reactions on PAH monomers (Fig. 1a) and soot surface (Fig. 1b). As shown in Fig. 1a, a PAH monomer was placed in the box center. The initial orientation and vibrational energy of PAH monomers were randomly sampled from the equilibrium configuration at the corresponding temperature. The initial distance between $\mathrm{H}$ atom and the center of mass $(\mathrm{COM})$ of PAH monomers is defined as $d=$ $\sqrt{x^{2}+b^{2}}$, where $x$ is set to $35 \AA$ and $b=\xi^{1 / 2} b_{\max }$ is the impact parameter [23]. $\xi$ is a random number that follows a uniform distribution in $(0,1)$, and $b_{\max }$ is the maximum impact factor in the sampling process, which is $6 \AA, 8.5 \AA$ and $10.5 \AA$ for A1, A7 and A19, respectively. The initial velocity of $\mathrm{H}$ atoms in $x$ direction was sampled from Maxwell-Boltzmann distribution at the sampled temperature, while velocity components in both $y$ and $z$ directions were set to 0 .

The second model represents the quasi-surface of soot particles. As shown in the left panel of Fig. $1 \mathrm{~b}$, the quasi-surface is composed of A7 or A19 monomers, and the minimum unit contains two columns of PAH stacks, which is built from five monomers. Periodic boundary conditions were applied to both $x$ and $y$-direction. In current configurations, the surface hydrogen atoms are exposed to the gas species from the $z$-direction without carbon-rich regions. This is consistent with the geometry of nascent soot particles $[24,25]$. To guarantee the initial configurations sitting at the potential minimum (see Fig. S1 in the Supplementary Materials), parameter $d$ was set as $3.3 \AA$ for A7s and A19s, and the values $h$ of A7s and A19s were 10.2 and $15.1 \AA$, respectively (Fig. 1b). A bottom restrain was added to the lowest (in $z$ direction) layer of atoms to avoid surface evaporation at high temperatures and improve the sample 
efficiency. The bottom restrain is a common method in the studies of metal surface reaction [26,27]. Prior to production simulations, the potential impact of the bottom restrain was carefully examined in Fig. S2. The bottom restrain causes negligible impacts on the reaction probabilities and kinetic energy transfer.

The initial setup of QCT simulations using the quasi-surfaces (Fig. 1b) is different from that of PAH monomers. An $\mathrm{H}$ atom was placed $20 \AA$ above the surface, and both the $x$ - and $y$-positions were randomly sampled. The initial $x-, y$ - and $z$-velocity components of $\mathrm{H}$ atom were randomly assigned from a Gaussian distribution, however, the speed distribution followed the Maxwell-Boltzmann distribution at the sampled temperature. This randomly sampled $x$-, $y$-, $z$ - components of incident velocity ensure the incident angle covering the range of $0^{\circ}$ to $180^{\circ}$. In addition, the surface temperature was consistent with the sampled temperature.

\section{(a) Monomer}
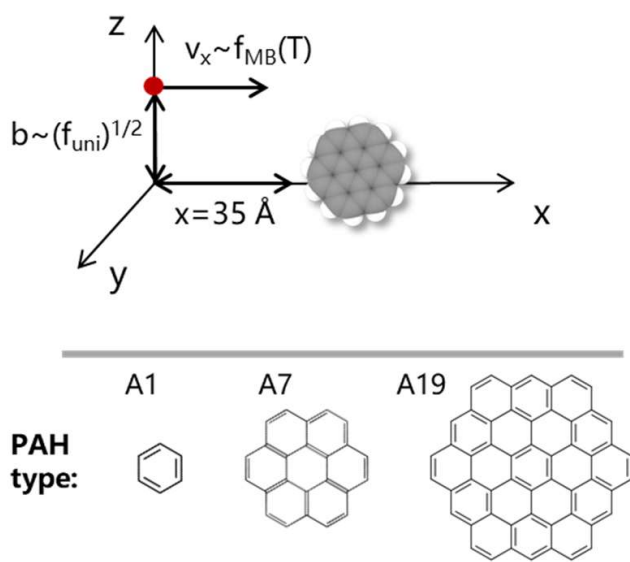

\section{(b) Quasi-surface}

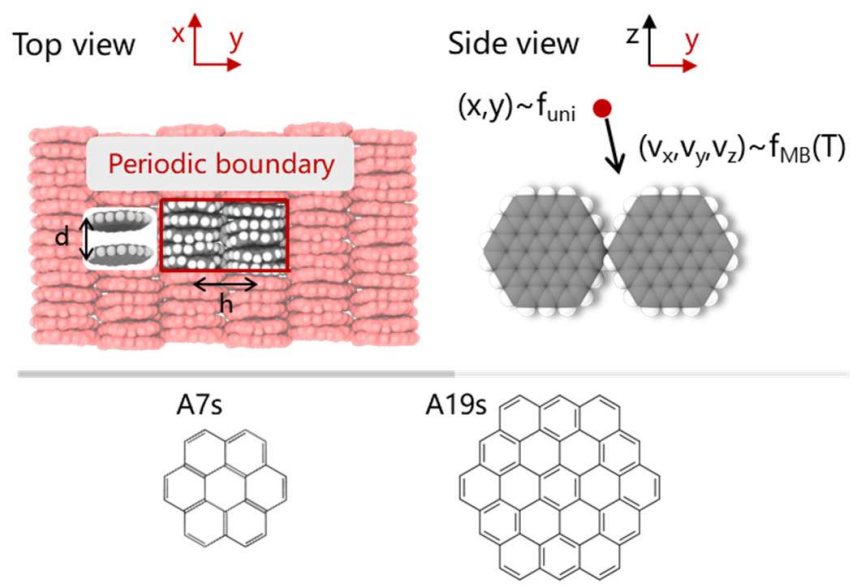

Figure 1. The initial configurations of quasi-classic trajectories for (a) PAH monomers and (b) PAH quasisurfaces. Periodic boundary conditions are applied to $x$ - and $y$-direction of PAH quasi-surface, and ghost molecules are represented by red color (top view in the left panel of (b)). $f_{M B}(T)$ and $f_{\text {uni }}$ represent the sampling from Maxwell-Boltzmann distribution and uniform distribution, respectively. $d$ and $h$ refer to the layer separation and distance between PAH stacks of the minimum unit in the models of quasi-surfaces. 
A temperature range of $1500-2700 \mathrm{~K}$ was selected to cover the flame conditions [28]. In this work, the temperature controls two main parameters, i.e., the relative velocity of incident $\mathrm{H}$ atom and the vibrational energies of PAH monomers and quasi-surfaces. The Nose-Hoover thermostat was applied for system equilibration (i.e., $10 \mathrm{ps}$ ), and the NVE ensemble was used for the further QCT simulations. At each temperature, the total number of trajectories was 6,000 and 3,000 for each PAH monomer and quasisurface, respectively. The selected numbers of trajectories were statistically sound to sample the reactivity of these configurations (Fig. S3). In total, 168,000 QCT simulations were conducted to reveal the reaction kinetics on PAH monomers and quasi-surfaces. All the trajectories were integrated using a time step of $0.2 \mathrm{fs}$ for a maximum time of $10 \mathrm{ps}$, and the system energy is conserved (Fig. S4). If the COM distance of two fragments exceeds $40 \AA$, the trajectory is truncated to avoid unnecessary calculations. All the MD simulations are carried out using LAMMPS simulator [29].

The collision cross section between $\mathrm{H}$ atom and PAH monomers can be written as

$$
\sigma_{\text {mono,H }}=\pi b_{\max }^{2}
$$

The collision cross section between $\mathrm{H}$ atom and quasi-surface $\left(\sigma_{\text {surf,H}}\right)$ is calculated by the geometric area of quasi-surface, which are $358.05 \AA^{2}$ and $514.91 \AA^{2}$ for $\mathrm{A} 7 \mathrm{~s}$ and $\mathrm{A} 19 \mathrm{~s}$, respectively. The reaction probability is defined as

$$
P_{r}=\frac{N_{r}}{N_{\text {total }}}
$$

where $N_{r}$ and $N_{\text {total }}$ are the number of reactions and total trajectories, respectively. The rate constant at the temperature $T$ is then calculated by

$$
k(T)=\left\langle v_{r}\right\rangle \sigma P_{r}
$$

with the mean collision velocity 


$$
\left\langle v_{r}\right\rangle=\left(\frac{8 k_{B} T}{\pi \mu}\right)^{\frac{1}{2}}
$$

where $k_{B}$ is the Boltzmann constant and $\mu$ is the reduced mass of reactants. The fraction of reaction on a reactive site $i$ is defined as the ratio of reaction events on site $i\left(N_{r, i}\right)$ to total reaction events $\left(N_{r}\right)$ at a temperature $T$,

$$
f_{i}(T)=\frac{N_{r, i}}{N_{r}}
$$

We can further have the per-site rate constant of site $i$ as

$$
k_{i}(T)=f_{i}(T) \times k(T) .
$$

\subsection{Force field validation}

The potential energy surface of PAH systems was explored using reactive molecular dynamics. We selected the ReaxFF force field to describe the interactions between carbon and hydrogen atoms, and the parameters were taken from Mao et al. [11,30], which has been widely used to describe the nucleation and growth of incipient soot particles. The ReaxFF parameters have been validated by the binding energy of PAH dimmers against the M06-2X/def2SVP level of theory [31]. To further examine the accuracy of ReaxFF force field on the collision dynamics between PAH and $\mathrm{H}$ atom, the intermolecular potential energy for benzene and $\mathrm{H}$ atom were calculated by the ReaxFF force field and a DFT method, that is, B3LYP-D3/6-311++G(d,p). Several data points from the calculations of CCSD(T)/cc-pVTZ are also added for reference. In Fig. 2, the energy curves are presented for four orientations. It is found that the results of ReaxFF force field are in good agreement with those using the B3LYP-D3/6-311++G(d,p) method. Comparing with the CCSD(T)/cc-pVTZ method, both ReaxFF and B3LYP-D3/6-311++G(d,p) methods overestimate the potential well depth by $\sim 0.43 \mathrm{kcal} / \mathrm{mol}$ at maximum. An unexpected fluctuation around $3.3 \AA$ exits in the prediction of ReaxFF forcefield in Fig. 2d. This indicates a potential issue in the 
original parametrization of the ReaxFF force field, but the fluctuation is less than $0.2 \mathrm{kcal} / \mathrm{mol}$ and does not influence the collision dynamics of $\mathrm{H}$ atom with PAHs in this study. More importantly, the ReaxFF force field is $\sim 150,000$ times faster than DFT method at the M06-2X/def2SVP level of theory [31], which enables the simulations of quasi-surface models.

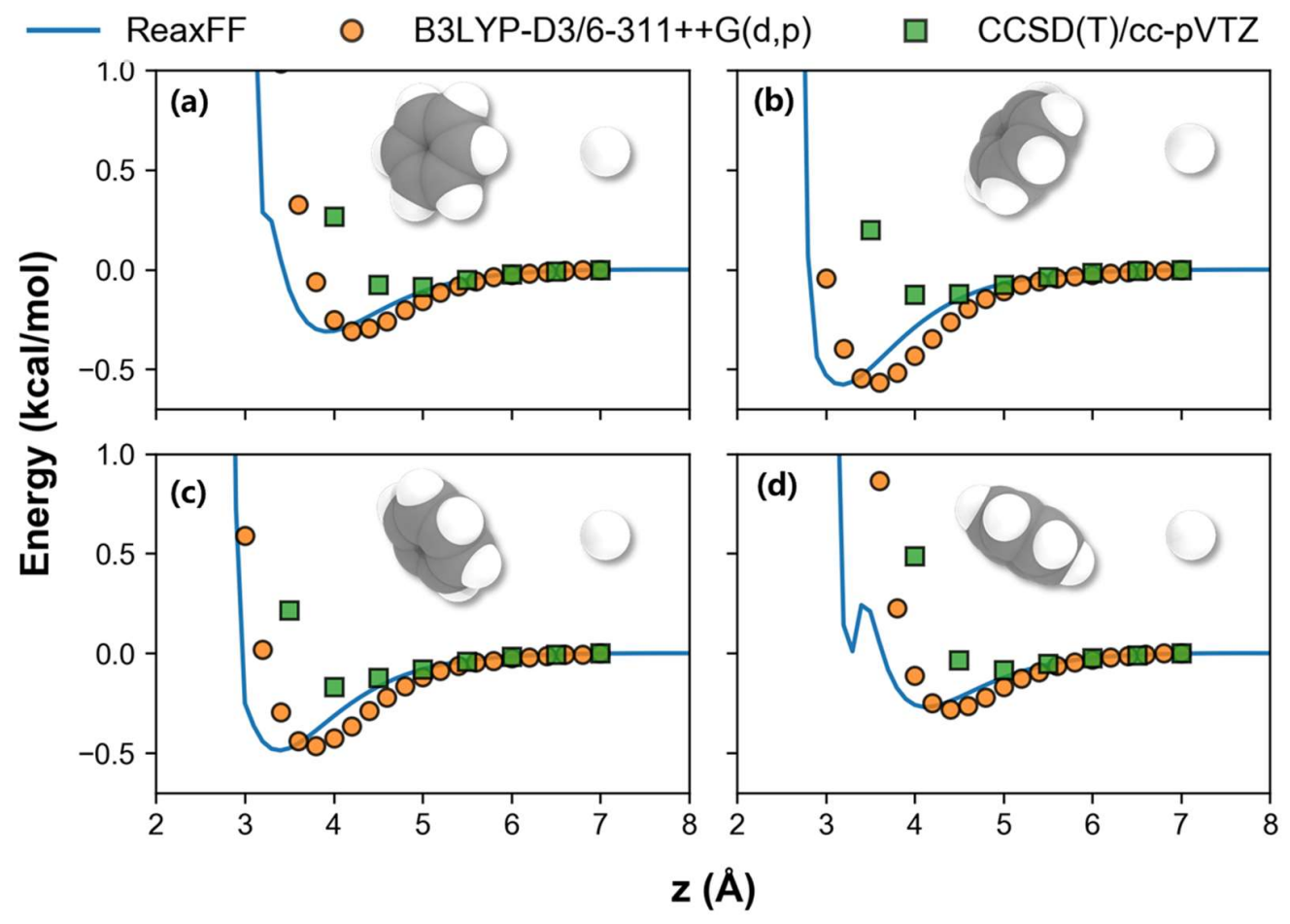

Figure 2. Intermolecular energy curves of a benzene with an $\mathrm{H}$ atom calculated from the methods of ReaxFF force field, B3LYP-D3/6-311++G(d,p) and CCSD(T)/cc-pVTZ.

\subsection{Post-process methods}

Solvent-excluded surface (SES) was used to evaluate the surface properties of PAH monomers and quasi-surfaces. This approach has been successfully applied to investigate surface reactivity of soot [14,15]. The SES area was calculated by MSMS 6.2.1 program [32] using a "rolling ball" algorithm. The radii of carbon and hydrogen atoms were taken from their van der Waals radii as $1.7 \AA$ and $1.2 \AA$, respectively [33]. The probe size was taken from the radius of hydrogen atom. For each case, 200 equilibrium structures were sampled from trajectories over $10 \mathrm{ps}$ at a specific temperature to calculate the 
mean and standard deviation values of SES area. In addition, the MSMS program has been updated to adapt periodic boundary conditions for the quasi-surface cases.

The collisions between atoms were determined by the van de Waals radii. The incident $\mathrm{H}$ atom can react with both $\mathrm{C}$ and $\mathrm{H}$ atoms on target configurations. A homemade code was developed to recognize the bond formation by the atomic distance and duration. If the distance of two atoms, i.e. $r$, is shorter than the critical value $r_{\text {crit }}$ and the duration exceeds $t_{\text {crit }}$, these two atoms are considered as "bonded". A detailed analysis has been performed to examine the effects of $r_{c r i t}$ and $t_{c r i t}$ on the predicted bonded information (Fig. S5). As a result, $r_{\text {crit }}$ and $t_{\text {crit }}$ is $1.2 \AA$ and 0.2 ps for C-H bonds, respectively. By contrast, we selected $r_{\text {crit }}=1.0 \AA$ and $t_{\text {crit }}=0.2$ ps for H-H bonds. The selected $r_{\text {crit }}$ values of C-H and $\mathrm{H}-\mathrm{H}$ bonds are comparable to their bond length in typical molecules; the bond length of $\mathrm{C}-\mathrm{H}$ bond in $\mathrm{C}_{6} \mathrm{H}_{6}$ is $1.083 \AA$ (calculated at the $\mathrm{CCSD}(\mathrm{T}) / \mathrm{cc}-\mathrm{pVTZ}$ level of theory) [34], and the bond length of H-H bond in $\mathrm{H}_{2}$ is $0.743 \AA$. Considering bond vibrations at high temperatures, $r_{c r i t}$ is longer than the bond length as expected. The bootstrapping resampling method [35] was used to extract error bars in our calculations. The sample size and the number of repeats is adapted as 3000 and 20, respectively (Fig. S6).

\section{Results and Discussion}

\subsection{Molecule structures}

Table 1. Surface properties of all considered configurations at 1500 and $2700 \mathrm{~K}$.

\begin{tabular}{lccccc}
\hline Case & $T(\mathrm{~K})$ & $A_{S E S}\left(\AA^{2}\right)$ & $\alpha_{H}$ & $R_{C, S E S}(\AA) R_{C, g e o}(\AA)^{\mathrm{a}}$ \\
\hline \multirow{2}{*}{ A1 } & 1500 & 106.03 & 0.49 & 2.90 & 3.68 \\
& 2700 & 108.61 & 0.49 & 2.94 & - \\
\multirow{2}{*}{ A7 } & 1500 & 273.38 & 0.38 & 4.66 & 5.99 \\
& 2700 & 275.44 & 0.36 & 4.68 & - \\
\multirow{2}{*}{ A19 } & 1500 & 496.38 & 0.29 & 6.28 & 8.40 \\
& 2700 & 502.77 & 0.28 & 6.33 & -
\end{tabular}




$\begin{array}{llllll} & 1500 & 475.72^{\mathrm{b}} & 0.79 & - & - \\ & 2700 & 471.26^{\mathrm{b}} & 0.75 & - & - \\ \mathrm{A} 19 \mathrm{~s} & 1500 & 767.03^{\mathrm{b}} & 0.82 & - & - \\ & 2700 & 786.52^{\mathrm{b}} & 0.77 & - & -\end{array}$

${ }^{\mathrm{a}} R_{c, \text { geo }}$ values are calculated using the optimized structure at the M06-2X/6-311G(d,p) level of theory.

${ }^{\mathrm{b}}$ The top surface of the minimum unit is reported here.

Table 1 lists the surface properties of PAH monomers and quasi-surfaces at 1500 and $2700 \mathrm{~K}$. The percentage of surface hydrogen is defined as $\alpha_{H}=A_{H} / A_{\text {total }}$, where $A_{H}$ and $A_{\text {total }}$ is the SES area of hydrogen atom and all surface sites, respectively. The $\alpha_{H}$ also refers to the probability of a successful collision on the surface $\mathrm{H}$ atoms. Note that both $A_{S E S}$ and $\alpha_{H}$ present weak dependence on the temperature, for example, the $A_{S E S}$ of $\mathrm{A} 19 \mathrm{~s}$ increases by only $\sim 2 \%$ when changing temperature from 1500 to $2700 \mathrm{~K}$. At $1500 \mathrm{~K}$, A1 yields the largest $\alpha_{H}$ as 0.49 , while the $\alpha_{H}$ of A19 is only 0.29 . This change can be attributed to the increase in the $\mathrm{C} / \mathrm{H}$ ratio from $\mathrm{A} 1$ to $\mathrm{A} 19$. As shown in Fig. 1b, the current configurations of quasi-surface models represent a hydrogen-rich surface as the surface $\mathrm{H}$ atoms point outward resulting in a large $\alpha_{H}$ as $\sim 0.80$ at $1500 \mathrm{~K}$, and the difference between A7s and A19s is minor, i.e. 0.02-0.03. The equivalent collision radius $R_{C, S E S}$ of PAH monomers can be calculated from SES area assuming spherical molecules, $R_{C, S E S}=\sqrt{A_{S E S} /(4 \pi)}+R_{H}$, where $R_{H}$ is the van der Waals radius of hydrogen atom as $1.2 \AA$. The geometrical radius $R_{\text {geo }}$ is extracted from the optimized structure at the M06-2X/6-311G(d,p) level of theory. The $R_{c, g e o}$ is calculated as

$$
R_{c, g e o}=d_{H, C O M}+R_{H}
$$

where $d_{H, C O M}$ represents the distance between the farthest $\mathrm{H}$ atom in a PAH to the COM. As Table 1 shows, $R_{c, g e o}$ values are always higher than $R_{c, S E S}$. Increasing the PAH size, the discrepancy between $R_{c, g e o}$ and $R_{C, S E S}$ increases from $\sim 0.78$ to $2.12 \AA$. 


\subsection{Collision dynamics}

PAH molecules are usually planar species [22], and the collision between PAH molecule and gaseous species depends on the relative orientation and velocity. We first examine the effect of orientation. Figure 3a shows that the impact factor reaches the maximum value $\left(b_{\max }\right)$ when the relative velocity is perpendicular to the plane of PAH molecules. When the relative velocity is parallel to the plane, the impact factor decreases to its minimum value. The collision status of all A1 trajectories is plotted in Fig. 3b. All 7 temperature cases from 1500 to $2700 \mathrm{~K}$, i.e. 42,000 trajectories, are included, and the collision status is tagged as "Collide" and "Miss". Clearly, all incident $\mathrm{H}$ atoms collide on PAHs when $b<3 \AA$, and the proportion of "Collide" trajectories gradually decrease from 1 to 0 when $3 \AA<b<6 \AA$. In this transition region, the relative orientation between the $\mathrm{PAH}$ molecule and $\mathrm{H}$ atom determines the collision dynamics. The relative velocity of $\mathrm{H}$ and $\mathrm{PAH}$ has a minor impact on the calculated collision probability $\left(P_{\text {coll }}\right)$ from 1500 to $2700 \mathrm{~K}$ due to the weak van der Waals potential (Fig. S7). It was reported that collisions between large particles at high temperatures could cause a significant reduction in the impact factor [36]. But in this work, the potential between PAH monomer and $\mathrm{H}$ atom is relatively weak that causes negligible impacts on collision events. The $P_{\text {coll }}$ values of A7 and A19 are included in Fig. S8, which are consistent with that of $\mathrm{A} 1$ (Fig. 3b). The collision radius from MD trajectories $\left(R_{c, M D}\right)$ can be calculated using

$$
R_{c, M D}=\left(\int_{0}^{b_{\max }} 2 b P_{\text {coll }}(b) d b\right)^{\frac{1}{2}}
$$

The derivation of $R_{c, M D}$ is included in the supplementary materials. Figure $3 \mathrm{c}$ shows that $R_{c, M D}$ is independent on the system temperature, but increases with PAH size as expected. The $R_{c, S E S}$ and $R_{c, g e o}$ values are also mapped in Fig. $3 \mathrm{c}$. It is clear that the computed $R_{C, S E S}$ and $R_{C, M D}$ are very close; $R_{c, S E S}$ overestimates the collision radius by $\sim 0.5 \AA$ in the worst case, i.e., A19. By contrast, $R_{c, g e o}$ always 
exceeds collision radius by at least $1 \AA$, and the maximum deviation is seen in A19 as $2.5 \AA$. The collision radius of PAH monomers can be well captured using $R_{C, S E S}$ without detailed MD trajectories.
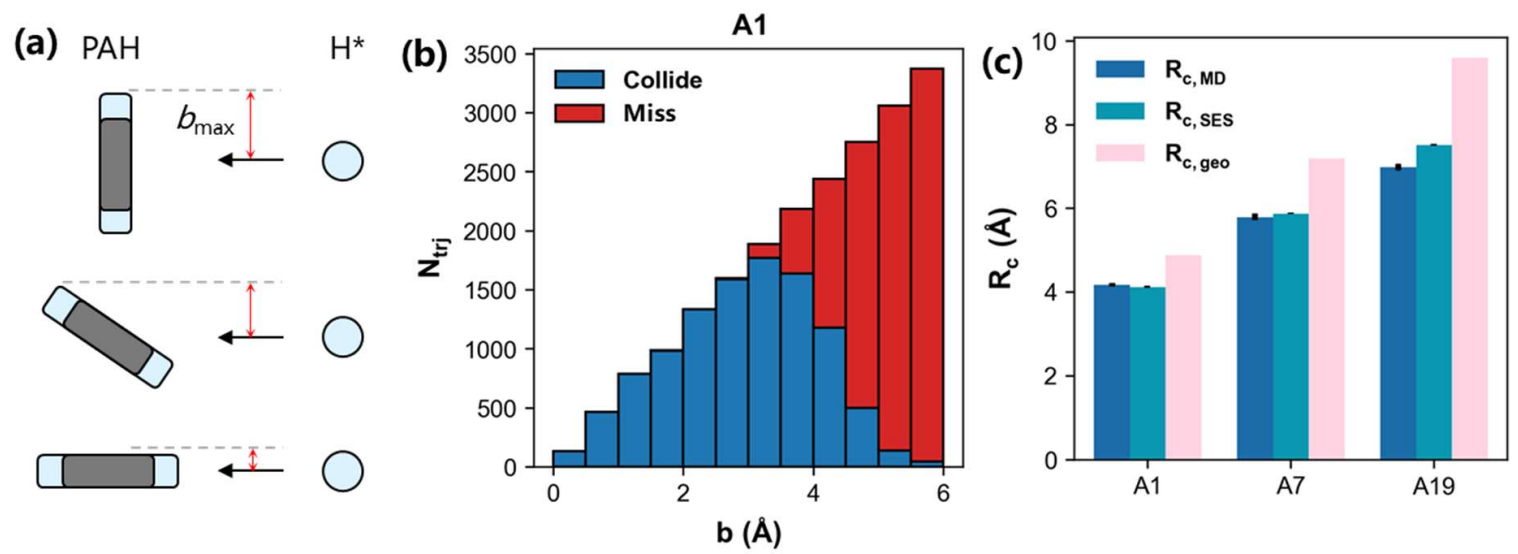

Figure 3. (a) Illustration of the impact factor in different orientations. (b) The status of collision trajectories between $\mathrm{A} 1$ and the incoming $\mathrm{H}$ atom with different impact factors. (c) The effective collision radius calculated by MD trajectories $\left(R_{c, M D}\right)$, SES area $\left(R_{c, S E S}\right)$ and geometrical radius $\left(R_{c, g e o}\right)$ of $\mathrm{A} 1, \mathrm{~A} 7$ and A19. The $R_{C, S E S}$ and $R_{C, M D}$ are the mean values taken from 1500 to $2700 \mathrm{~K}$, and the error bars represent one standard deviation.

The potential reaction dynamics between PAH monomer and $\mathrm{H}$ atom was then discussed in detail. Two types of reactions were observed including hydrogen abstraction (HB) reactions and hydrogen addition (HD) reactions. In an $\mathrm{HB}$ reaction, an $\mathrm{H}$ atom is abstracted from a $\mathrm{PAH}$ by the incident $\mathrm{H}$ to form a $\mathrm{H}_{2}$ as the product, which plays an important role in the processes of PAH growth and soot formation. In an $\mathrm{HD}$ reaction, the incident $\mathrm{H}$ atom directly bonds with a $\mathrm{C}$ atom on a PAH. Compared with $\mathrm{HB}$ reactions, the HD reaction is less studied in the field of soot, but it is common in the interstellar PAHs of astrochemistry [37], where the temperature is much lower than sooting flames. Figure $4 \mathrm{a}$ shows the probability $\left(P_{H B}\right)$ of $\mathrm{HB}$ reactions as a function of temperature for $\mathrm{A} 1, \mathrm{~A} 7$ and $\mathrm{A} 19$. For all the PAH molecules, $P_{H B}$ increases monotonically with temperature as expected in Fig. 4a. HB reactions occur 
more easily on the smaller PAH; A1 molecule yields the highest $P_{H B}$ as 0.023 at $2700 \mathrm{~K}$, while the $P_{H B}$ of A19 molecule is only 0.009 . This can be attributed to the surface properties that a larger PAH molecule has a lower $\alpha_{H}$, leading to a lower reaction probability.

The corresponding reaction rate constants calculated from Eq. 3 are also listed in Fig. 4b. At high temperature cases $(>2100 \mathrm{~K}), \mathrm{A} 1, \mathrm{~A} 7$ and A19 share similar reaction constants, while A19 yields lower reaction constants by $50 \%$ at temperatures lower than $2300 \mathrm{~K}$ compared to both $\mathrm{A} 1$ and $\mathrm{A} 7$. These inconsistent rate constants between small and large PAHs raise a critical argument whether the rate constants extracted from small PAHs can be used for large ones. Our results in Fig. 4b provide a failure of the above argument. The accurate rate constants of $\mathrm{HB}$ reactions on A1 molecules are estimated from TST calculations in previous works, for example, Hou et al. [7] and Liu et al. [8]. In general, our predicted rate constants of A1 from QCT method are in line with that of Liu et al. [8] at the CBS-QB3 level of theory except the higher ones at 1500-1900 K. Hou et al. [7] estimated the HB rate constants of A1 from the M06-2X/6-311G(d,p) method, which was benchmarked against the CCSD(T)/CBS level of theory. Our QCT results are close to the upper bound of Hou et al. [7] considering the uncertainties (i.e. shadow region). Rate constants from mass-spectrometric measurements by Park et al. [38] are also plotted in Fig. 4b. When comparing with rates by DFT and QCT methods, the experimental measurement seems to underestimate the rates of HB reaction. Again, at 1500-1900 K, QCT overestimates the rate constants by $50 \%$. 

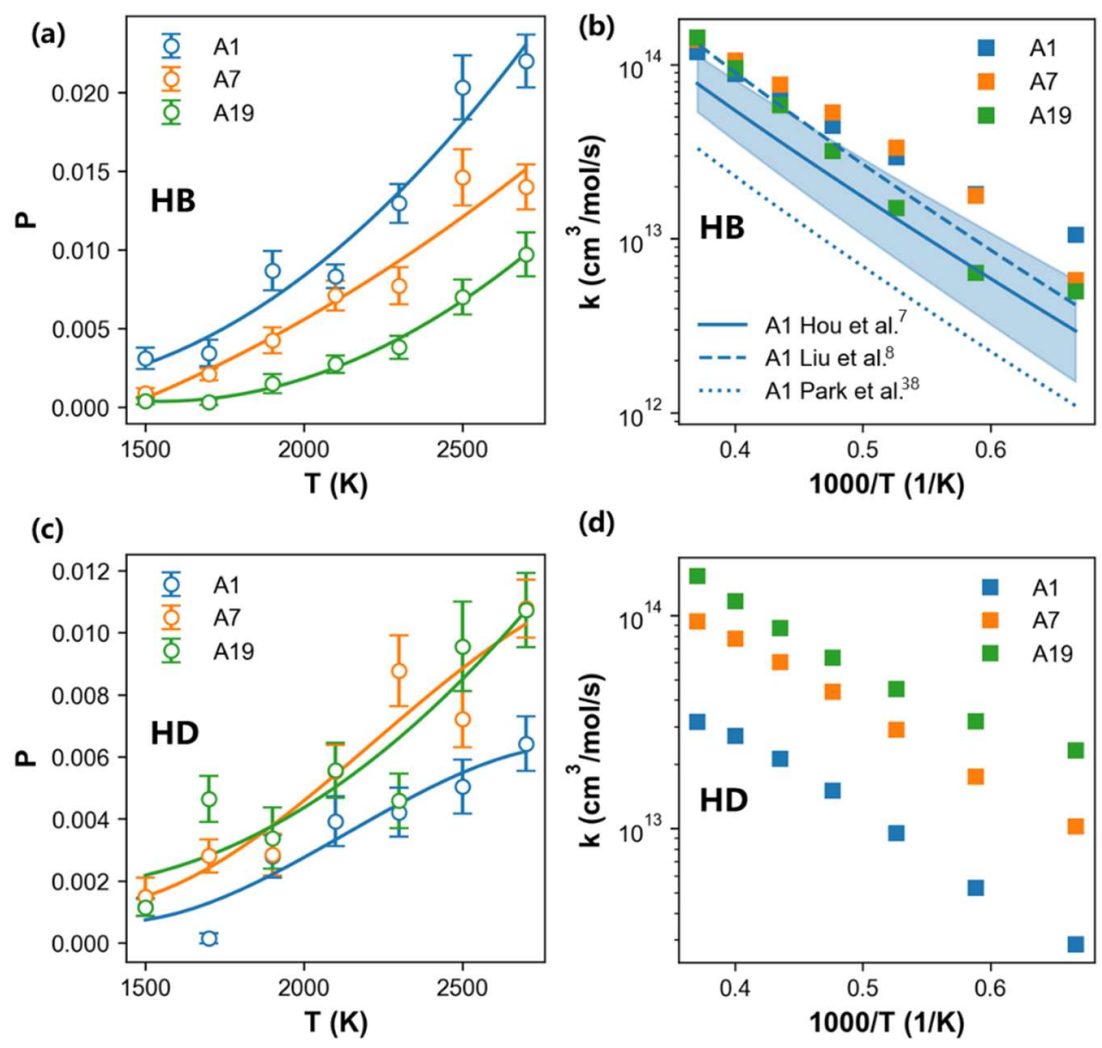

Figure 4. Reaction probabilities of (a) hydrogen abstraction and (c) hydrogen addition for A1, A7 and A19 from 1500 to $2700 \mathrm{~K}$, and the corresponding rate constants for (b) hydrogen abstraction and (d) hydrogen addition. The curves are fitted using a polynomial function. The rate constants are extracted from the fitted curves. The shadow region in (b) represents the errors estimated at the M06-2X/6-311G(d,p) level of theory [7]. The dash curve in (b) represents the rate constants taken from the CBS-QB3 level of theory [8]. The dot curve in (b) represents the rate constants taken from the mass-spectrometric measurements [38].

The trend of HD reactions does not follow that of $\mathrm{HB}$ reactions. In Fig. 4c, the HD reaction probabilities $\left(P_{H D}\right)$ of A7 and A19 are about 2 times larger than that of A1. Although an A19 molecule has more edge carbons for potential addition reactions, its $P_{H D}$ are not higher than that of A7. Because not all edge carbons could react with the incident $\mathrm{H}$ atom. The effect of site type will be discussed in the latter section. Note that the slope of $P_{H D}$ gradually decreases as the temperature approaches $2700 \mathrm{~K}$ in 
the case of A1. In a recent study of ring polymer molecular dynamics for $\mathrm{H}$ sticking on graphene surface [39], it was found that the sticking probabilities of $\mathrm{H}$ atom drops at high incident kinetic energies. In other words, $P_{H D}$ does not increase monotonically with the incident kinetic energy, but decreases after reaching a critical one. This phenomenon is also observed in other systems [40,41]. For example, Valentini et al. [41] reported that the sticking probability of $\mathrm{O}_{2}$ on $\mathrm{Pt}$ (111) surface reaches its local maximum with a critical incident kinetic energy $\left(E_{i}\right)$ as $0.4 \mathrm{eV}$. When $E_{i}>0.4 \mathrm{eV}$, the sticking probability of $\mathrm{O}_{2}$ begins to decrease because $\mathrm{O}_{2}$ can easily escape from the chemisorbed state $(-0.25 \mathrm{eV})$. The critical kinetic energy is affected by the local potential energy surface and the energy transfer in the collision. In this work, the $P_{H D}$ of A1 remains monotonical until $2700 \mathrm{~K}$. For larger PAHs, the interaction becomes stronger causing an increase in the critical kinetic energy. As a result, the critical incident kinetic energy increases with PAH size.

Figure $4 \mathrm{~d}$ presents the rate constants of HD reactions on A1, A7 and A19; clearly, the reaction constant is proportional to the PAH size. More importantly, it is found that the rate constants of HD reactions on A19 are comparable with those of $\mathrm{HB}$ reactions, for example, the rate constant of HD reaction on A19 is three times larger than that of HB reactions at $1900 \mathrm{~K}$. By contrast, A1 undergoes slower HD reactions, and at $1900 \mathrm{~K}$, the ratio between $\mathrm{HD}$ reactions and $\mathrm{HB}$ reactions is 0.31 . Again, this indicates the misuse of small PAHs to represent the large PAHs considering the underlying reaction kinetics. The channel of HD reactions might be largely underestimated in the community due to the knowledge derived from the small PAHs (i.e. A1), and the above observation highlights the significance of direct estimation of rate constants from large PAHs.

Next, we compared the HB/HD reaction probabilities between an $\mathrm{H}$ atom and quasi-surface models, i.e. A7s and A19s. The $P_{H B}$ of A7s and A19s (Fig. 5a) shows minor differences except at high temperatures, suggesting that the PAH size has a little effect on the reaction probability of the quasi- 
surface. This observation is expected due to the similar potential energy surface of A7s and A19s. The overall rate constants of $\mathrm{HB}$ reactions are shown in Fig. $5 \mathrm{~b}$; the rate constants of A19s are about 1.5 times higher than those of A7s due to larger collision cross section. Also, the slopes of the rate constant curves are similar for these two cases. It is thus learnt that the collision cross section plays a more significant role in the HB reaction compared to the type of PAHs. In Fig. 5c, the $P_{H D}$ of A7s shows a similar trend comparing with the reactions among A1 monomers, and the $P_{H D}$ values reach its maximum at $2500 \mathrm{~K}$. As discussed in Fig. 4c, the $P_{H D}$ decreases after reaching the critical incident kinetic energy (or temperature), and the critical incident kinetic energy increases with PAH size. As a result, the HD rate constant of A7s shows an obvious drop at high temperatures. Below $2500 \mathrm{~K}$, the difference in $P_{H D}$ between A19s and A7s is limited. Again, the HD rate constants of A19s are 2-3 times larger than those of A7s. Note that the quasi-surface model prefers abstraction reactions as the rate constants of HB reaction are larger than those of HD reactions in most cases. In other words, the abstraction reaction prevails in surface reactions unlike gas-phase reactions. 

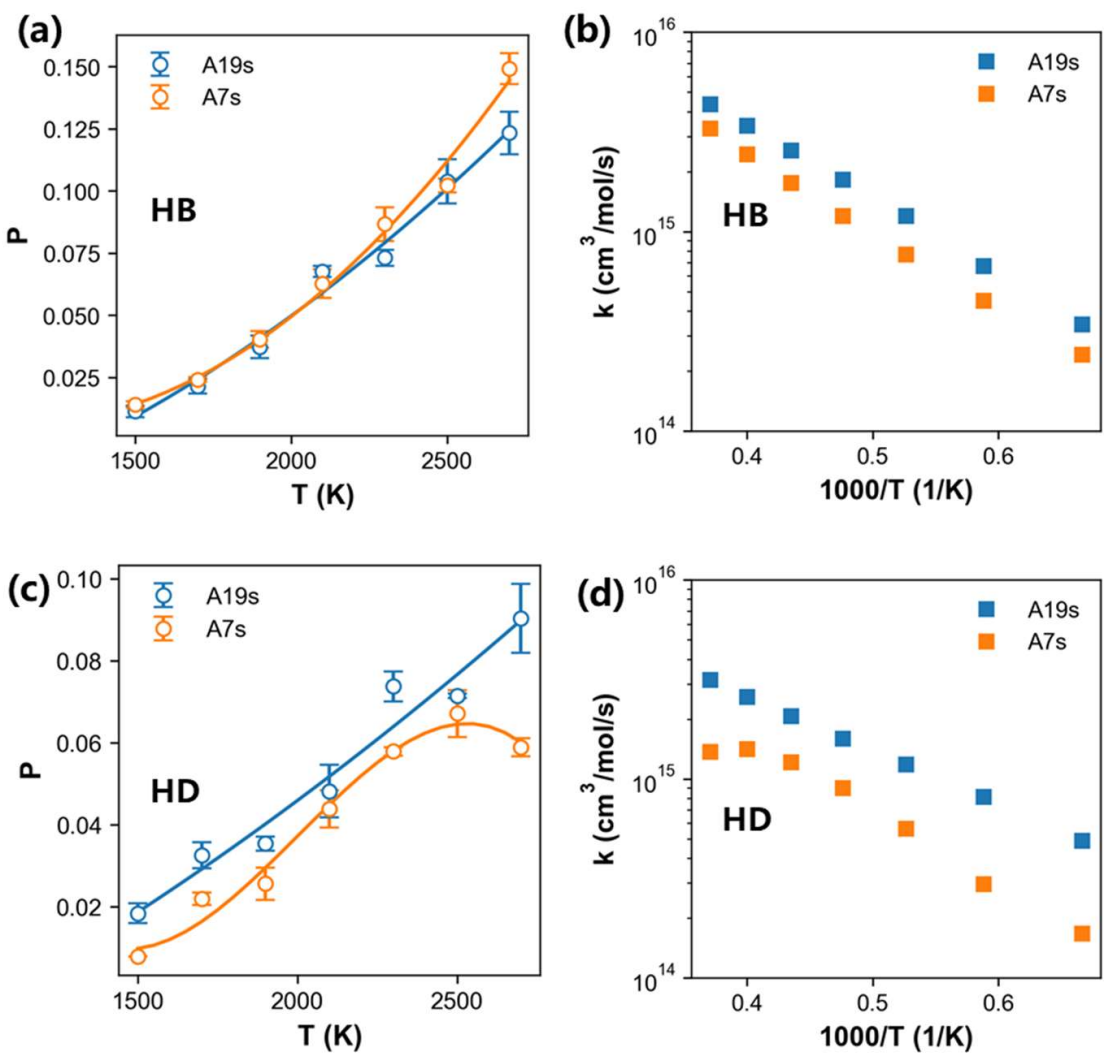

Figure 5. Surface reaction probabilities of (a) hydrogen abstraction and (c) hydrogen addition for A7s and A19s from 1500 to $2700 \mathrm{~K}$, and the corresponding rate constants for (b) hydrogen abstraction and (d) hydrogen addition. The curves are fitted using a polynomial function. The rate constants are extracted from the fitted curves.

\subsection{Site effect}

The overall reaction kinetics of both HB and HD reactions are decomposed into reactions on each type of reactive sites. The atoms on PAH monomers and quasi-surface models are classified into three site types, i.e., inner, zig-zag (ZZ) and free-edge (FE) site (Fig. 6b). The inner atoms cannot react with $\mathrm{H}$ atoms at 1500-2700 K, and thus they are excluded from our discussion here. In Fig. 6a and 6b, the site types on A7 and A19 monomers are highlighted; both A7 and A19 monomer has 12 FE sites, while A19 monomer also includes $6 \mathrm{ZZ}$ sites. In the quasi-surface models, the available sites depend on the local geometry. The reactive sites are further marked by their relative positions to the neighboring molecule in 
Fig. $6 \mathrm{c}$ and $6 \mathrm{~d}$. The corresponding SES area of each site are reported for monomers and quasi-surface models. In Fig. 6e, it is found that the surface areas of $\mathrm{H}$ atoms in the most FE sites are in the range of 8.26-8.96 $\AA^{2}$. However, atoms in FE-1 sites barely contribute to the surface area $\left(\sim 1.0 \AA^{2}\right)$ due to the hindrance effect. $\mathrm{H}$ atoms in $\mathrm{ZZ}$ sites occupy similar surface area regardless its relative position to the neighboring molecules. It is interesting to note that the surface area of carbon in monomers is about 4 times larger than that of carbon in quasi-surface models. This is consistent with the indication of $\alpha_{H}$ (Table 1) as the quasi-surface model is dominated by hydrogen atoms.
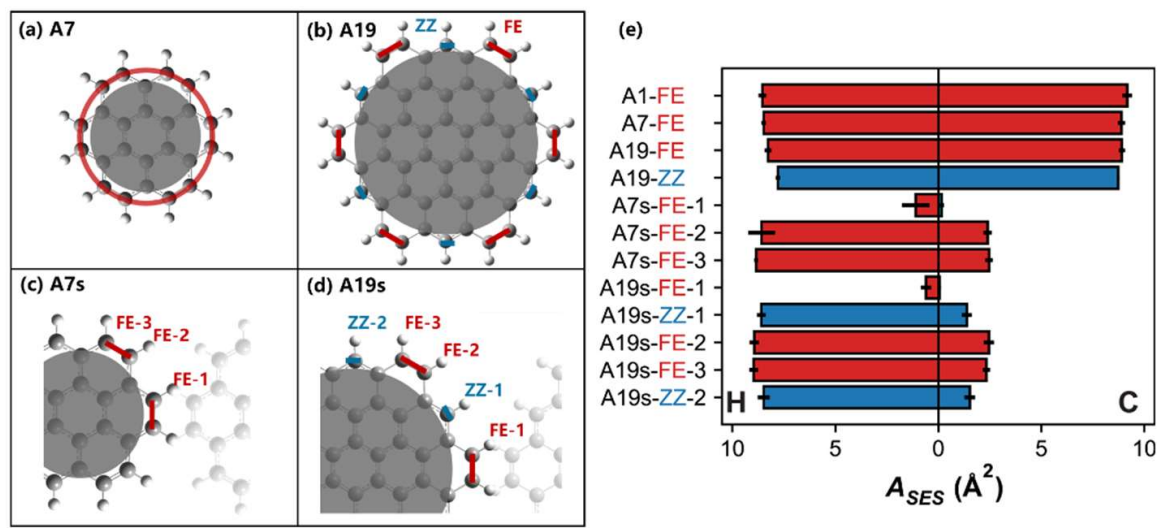

Figure 6. (a-d) The illustrations of site types in A7, A19, A7s and A19s. Red and blue lines represent freeedge site, and zigzag edge site, respectively. The grey transparent region represents inner $\mathrm{C}$ atoms. (e) The solvent-excluded surface area $\left(A_{S E S}\right)$ of hydrogen and carbon atoms in all sites. The error bars are extracted from the equilibrated trajectories of $10 \mathrm{ps}$ to present one standard deviation.

The incident angle of $\mathrm{H}$ atoms is a critical parameter determining the fate of collision. The sampled angles of incident velocities are in the range of $0-180^{\circ}$ as discussed in the previous section. In Fig. 7 , the incident angle governs the accessibility of surface sites in the first collision event considering the weak van der Waals potential (Fig. 1). In particular, the available incident angle of FE-3 site is up to145, while the accessibility of ZZ-1 site is limited as the available incident angle is about $54^{\circ}$. Here, the FE-3 and ZZ-2 sites are termed as "flat sites", which sit on the edge of quasi-surfaces allowing a wide range of 
incident angles. By contrast, FE-1, FE-2 and ZZ-1 sites are considered as "pocket sites", which has limited accessibility for gaseous species due to the geometry hindrance. The definition of pocket sites here is in line with the previous work [15]. It was assumed that pocket sites cannot be accessed by gaseous species and thus they were treated as unreactive sites. In this work, it would be interesting to resolve the question whether reactive sites in pocket are accessible in collision events.

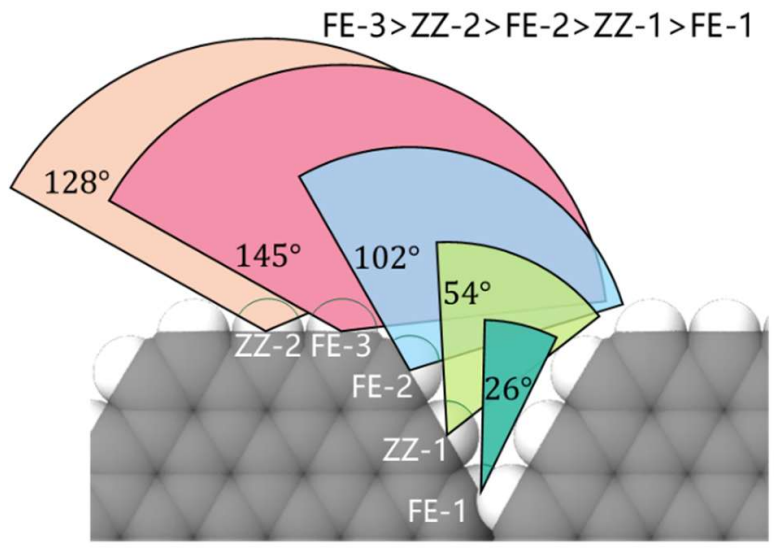

Figure 7. Illustration of available incident angles for each site on the A19s surface. The snapshot is taken from the $y-z$ plane.

QCT trajectories are further examined to count the numbers of collisions and reactions on each site type. For each case, the trajectories over all temperature ranges are analyzed $(42,000$ for each PAH monomer and 21,000 for each quasi-surface model). The results presented here refer to the per-site basis. The cases of A1 and A7 monomers are not included because they only have FE sites. $N_{\text {col,1st }}$ represents the count of collision events that the incident $\mathrm{H}$ first collides with $\mathrm{H}$ and $\mathrm{C}$ atoms on a particular reactive site (Fig. 8a-c). Note that each reactive site is composed of one $\mathrm{H}$ and one $\mathrm{C}$ atoms, the incident $\mathrm{H}$ could access both $\mathrm{C}$ and $\mathrm{H}$ atoms during the first collision due to the incident kinetic energy. Figure $8(\mathrm{a}-\mathrm{c})$ show that the surface $\mathrm{H}$ atoms and $\mathrm{C}$ atoms have roughly equal probability to be collided on in the first collision despite the difference in the surface area (Fig. 6e). For A19 monomers, the $N_{c o l, 1 s t}$ on $\mathrm{H}$ atoms of FE 
site exceeds that of $\mathrm{ZZ}$ site by $\sim 16 \%$, which is consistent with the small difference in the surface area between two sites. For quasi-surface models, the $N_{\text {col,1st }}$ of FE-1 site is much lower than other FE sites, which is 4.1 and 2.0 for $\mathrm{A} 7 \mathrm{~s}$ and A19s, respectively. These small values are expected considering their negligible contribution in the surface area (Fig. 6e) and the incident angle (Fig. 7). Taking a further looking at the comparison between FE-2 and FE-3 sites, the $N_{c o l, 1 s}$ values of $\mathrm{H}$ atoms on FE-3 site is $\sim 60 \%$ larger than of the FE-2 site highlighting the effect of incident angle, even though their $A_{S E S}$ values are the same. The above cases indicate the significance of surface area and incident angle in the determination of the overall collision rate.
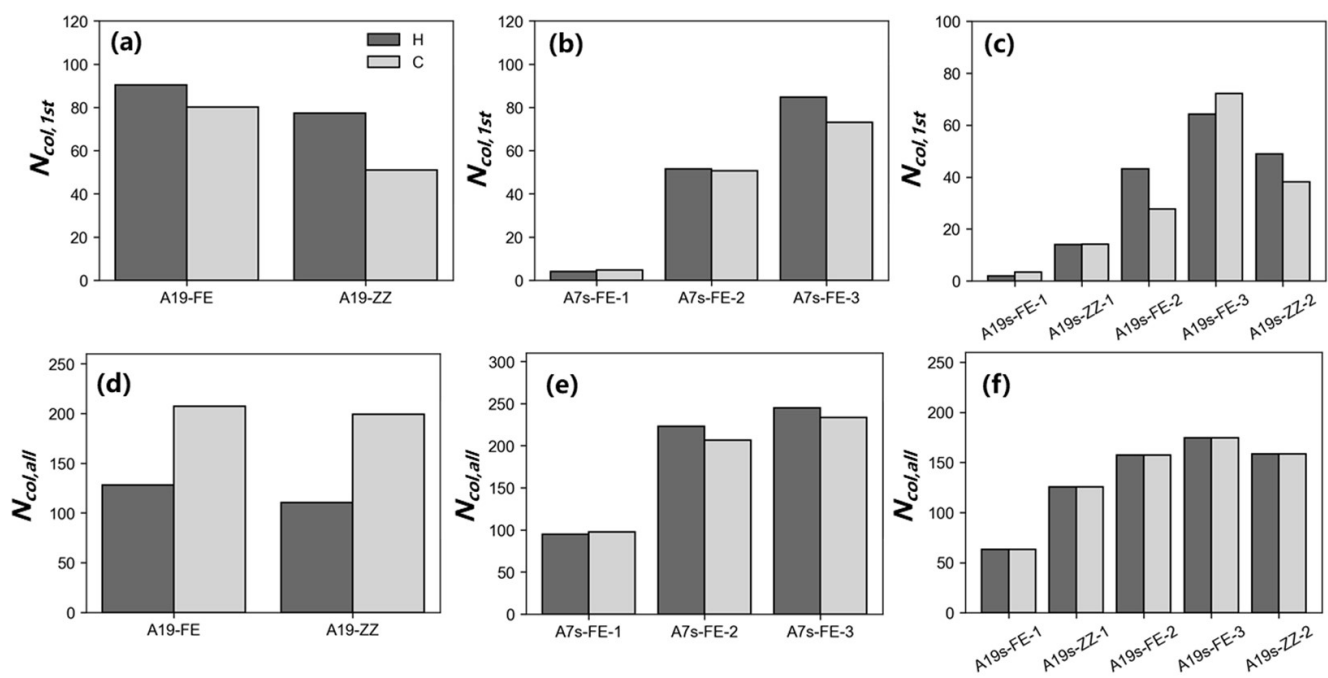

Figure 8. Numbers of collisions of each reactive site in the first collision event between the incident $\mathrm{H}$ atom and surface atoms on (a) A19, (b) A7s and (c) A19s. Numbers of collisions of each reactive site during the whole trajectory for cases of (d) A19, (e) A7s and (f) A19s. Number of collisions on H and C atoms are represented by dark-grey and light-grey, respectively.

After the first collision, the incident $\mathrm{H}$ atom might be absorbed on particle surface and visit other sites. In Fig. 8(d-f), the total number of collisions accessed by the incident $\mathrm{H}$ atom within a trajectory are termed as $N_{c o l, a l l}$ for each site type. For surface $\mathrm{H}$ atoms, the $N_{\text {col,all }}$ of PAH monomer and quasi- 
surface models is at least $40 \%$ and $170 \%$ larger than the corresponding $N_{c o l, 1 s t}$, respectively. In particular, the ratio of $N_{c o l, a l l} / N_{c o l, 1 s t}$ is up to 31 for the FE-1 site on A19s, and this highlights the local accumulation of the incident $\mathrm{H}$ atoms into the surface pocket. A similar trend is observed from surface $\mathrm{C}$ atoms as well.

In the classical gas-kinetic theory, collision modes are classified into the specular and diffusive scattering accounting for the momentum transfer [42-44]. It is well accepted that gaseous bimolecular reactions undergo a single collision leading to the product, and the unsuccessful collision results in a specular scattering. This underlying assumption underpins the majority of combustion kinetics in gaseous bimolecular reactions [45-48], and is further extrapolated in the treatment of surface reactions to an extent. However, the gas-nanoparticle scattering could exhibit a diffusive mode that the gas molecule is trapped on the particle surface due to the energy accommodation of nanoparticle. In previous works, the impact of diffusive scattering (termed as "surface diffusion" here) is well examined in terms of the transport properties of nanoparticles [49], but its potential effect on the surface reactions is largely neglected in the treatment of surface models $[6,50]$. To examine the potential impact of surface diffusion on the reactions, we define the number of reactions occurring on each site as $N_{\text {reax }}$. Figure 9 compares the correlation among $N_{c o l, 1 s}, N_{c o l, a l l}$ and $N_{\text {reax }}$. It is noted that $N_{\text {col,all }}$ has a better linear correlation with $N_{\text {reax }}$. This suggests that the first collided sites do not determine the overall reaction rate. We believe that the incident $\mathrm{H}$ atom can bounce on the surface and react on reactive sites rather than the first collided site. This phenomenon will be addressed in the later context. 

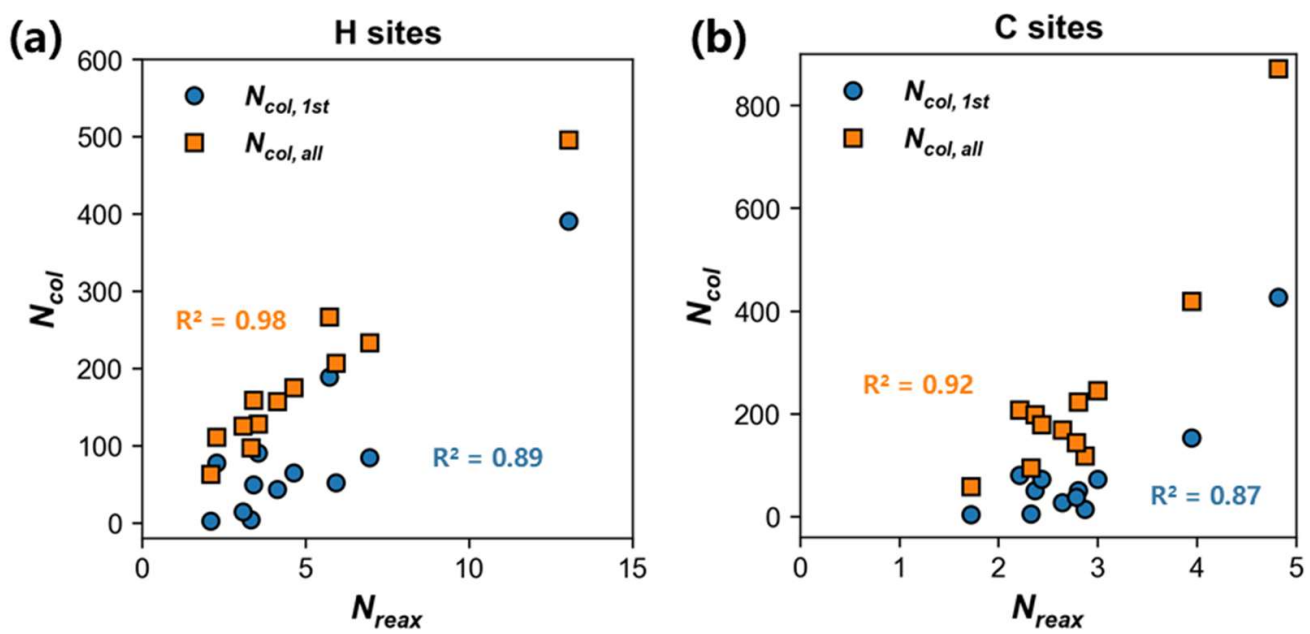

Figure 9. The correlation between the number of collisions and $N_{\text {reax }}$ for A1, A7, A19, A7s and A19s. The blue and yellow dots represent $N_{c o l, 1 s t}$ and $N_{c o l, a l l}$, respectively. The statistics are taken from all sites in Fig. 8. The correlation coefficients $\left(\mathrm{R}^{2}\right)$ between $N_{\text {reax }}$ and $N_{c o l, 1 s t}, N_{\text {reax }}$ and $N_{\text {col,all }}$ are also marked in the figure.

The per-site rate constants of HB and HD reactions on each site are presented in Fig. 10. All sites are divided into four sub-groups, i.e., surface FE, surface ZZ, surface FE (pocket) and sites in PAH monomers following the magnitude of rate constants of HB reactions. The rate constants of a particular site overlap with other sites in each sub-group. The per-site rate constants of HB reactions in monomers are compared with those at the CBS-QB3 level of theory [8]. The overall rate constants from Ref. [8] are converted to per-site rate constants by dividing the numbers of sites. The trend of FE sites on A1 is discussed in Fig. 4, which is not further elaborated here. As shown in Fig. 10a, the rate constants of HB reaction decrease with $\mathrm{PAH}$ size in the range of $\mathrm{A} 1, \mathrm{~A} 7$ and $\mathrm{A} 19$. The FE sites show higher reactivity than $\mathrm{ZZ}$ sites for HB reactions, e.g., rate constants of $\mathrm{HB}$ reactions on FE sites are $\sim 0.8$ times higher than those on $\mathrm{ZZ}$ sites at $2100 \mathrm{~K}$. Liu et al. [8] also included the reduced rate constant in phenanthrene (A3) compared to that in A1. This is consistent with our results that the rate constant of HB reactions is inversely proportional to the PAH size to some extent. Considering the site effect, the rate constants of P3-CS1 site (similar to FE 
site in this paper) on A3 is $\sim 20 \%$ larger than those of P4-CS1 site (similar to ZZ site in this paper) [8]. Again, this is in line with our prediction that free edge exhibits higher reactivity for HB reactions.
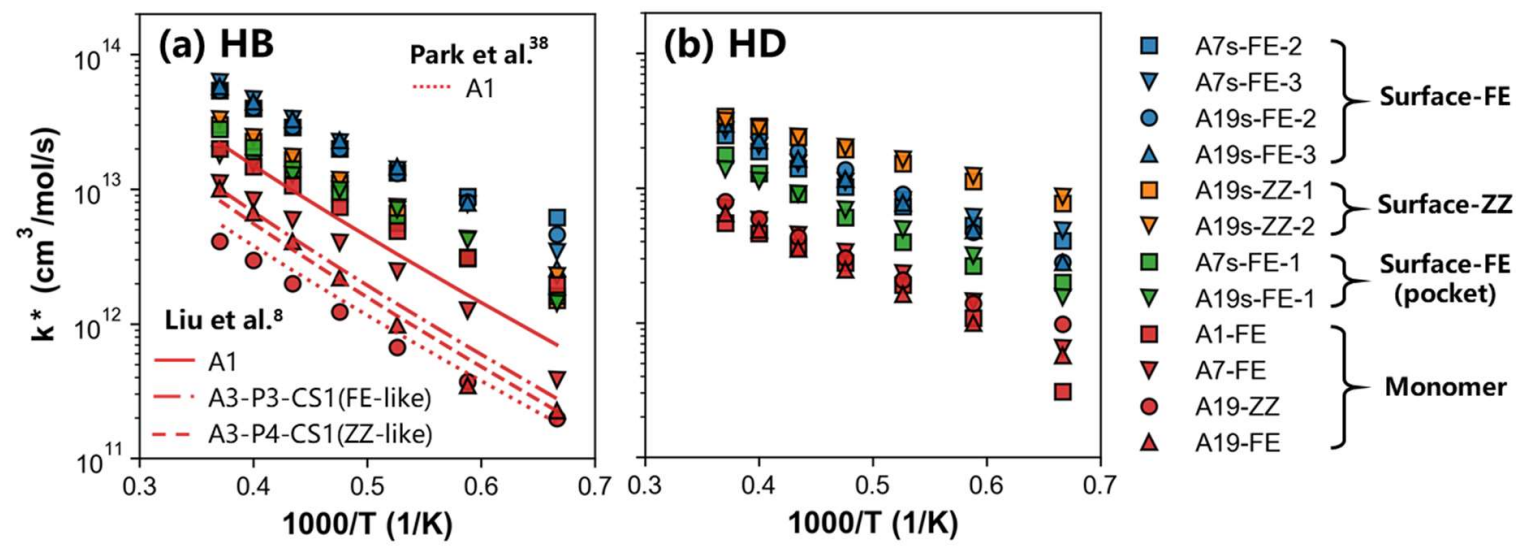

Figure 10. Site-specific rate constants of (a) hydrogen abstraction and (b) hydrogen addition reactions on PAH monomers and quasi-surfaces. Four groups of sites, that is, surface-FE, surface-ZZ, surface-FE (pocket) and sites in PAH monomers are represented by blue, orange, green and red markers, respectively. Rate constants of A1-FE, A3-P3-CS1 and A3-P4-CS1 calculated by Liu et al. [8] are also represented by solid line, dash line and dash-dot line, respectively.

Figure 10 includes all rate constants on each site in this work. The first three sub-groups include sites on the quasi-surface, and the last group refer to sites in PAH monomers. It is noticed that the sites in the quasi-surface models have much higher reaction rates for HB reactions than the sites in PAH monomers considering the impact of surface diffusion. For example, the rate constants on A19s-FE-2 sites are almost one order of magnitude higher than those on A19-FE sites. The rate constants (Fig. 10a) of FE-2 and FE3 sites are nearly identical on both quasi-surface models. This can be attributed to the balance of the available incident angle and the local potential energy surface; reactive sites in the flat region (FE-3) have better exposure to the gaseous species, that is, larger $A_{S A S}$ and available incident angle, while FE-2 sites sit next to the pocket region and exhibit a stronger local attraction. Regardless the impact of stronger attraction, the rate constants of FE-1 sites are $\sim 2.3$ times lower than those of FE-2 and FE-3 on average 
due to the minimal $A_{S E S}$. This supports the previous assumption [15] that the reactive sites in the pocket yield reduced rates due to geometry hinderance. The rates of HD reactions on quasi-surface are also enhanced via the event of surface diffusion (Fig. 10b). Unlike HB reactions, HD reactions prefer surface $\mathrm{ZZ}$ sites rather than surface FE sites by a factor of 1.81 at $1900 \mathrm{~K}$.

Table 2. Per-site rate constants for hydrogen abstraction and addition reactions on surface. ${ }^{\mathrm{a}}$

\begin{tabular}{lcccc}
\hline \multirow{2}{*}{ Site type } & \multicolumn{2}{c}{$\mathrm{HB}$} & \multicolumn{2}{c}{$\mathrm{HD}$} \\
\cline { 2 - 5 } & $\mathrm{A}\left(\mathrm{cm}^{3} / \mathrm{mol} / \mathrm{s}\right)$ & $\mathrm{E}_{\mathrm{A}}(\mathrm{kcal} / \mathrm{mol})$ & $\mathrm{A}\left(\mathrm{cm}^{3} / \mathrm{mol} / \mathrm{s}\right)$ & $\mathrm{E}_{\mathrm{A}}(\mathrm{kcal} / \mathrm{mol})$ \\
\hline Surface-FE & $2.0 \times 10^{15}$ & 17.70 & $3.03 \times 10^{6} \times \mathrm{T}^{2.204}$ & 5.94 \\
Surface-FE (pocket) & $8.6 \times 10^{14}$ & 17.80 & $2.04 \times 10^{4} \times \mathrm{T}^{2.719}$ & 3.97 \\
Surface-ZZ & $1.5 \times 10^{15}$ & 19.20 & $1.44 \times 10^{6} \times \mathrm{T}^{2.292}$ & 5.61 \\
A1-FE & $3.4 \times 10^{14}$ & 15.70 & $4.89 \times 10^{6} \times \mathrm{T}^{1.891}$ & 5.42 \\
Surface $^{\mathrm{b}}$ & $4.67 \times 10^{9} \times \mathrm{T}^{1.656}$ & 16.50 & - & - \\
A1-FE & $5.38 \times 10^{9} \times \mathrm{T}^{1.582}$ & 15.67 & - & - \\
A1-FE & $6.75 \times 10^{8} \times \mathrm{T}^{1.910}$ & 15.61 & - & - \\
A1-FE & - & - & $4.03 \times 10^{13}$ & 4.31 \\
\hline
\end{tabular}

${ }^{a}$ The rate constants in this work are fitted into the expression of $k=A T^{n} \exp \left(E_{A} / R T\right)$.

${ }^{\mathrm{b}}$ Rate constants of HB reactions are taken from Hou et al. [7].

${ }^{c}$ Rate constants of HB reactions are taken from Liu et al. [8].

d Rate constants of HD reactions are taken from Nicovich and Ravishankara [51].

Table 2 summarizes all per-site rate constants in Arrhenius expressions recommended for kinetic models. We fitted the reaction constants of three sub-groups of sites including surface FE, surface FE (pocket) and surface ZZ. For the first two sub-groups, the activation energies are the same, but the preexponential factor of surface FE sites is $\sim 2.3$ times of the FE sites at the pocket highlighting their limited accessibility. Rate constants of surface HB reactions calculated by TST method from Hou et al. [7] and Liu et al. [8] are also listed in Table 2 for reference. The HD reactions are in the range of 3.97 to 5.94 $\mathrm{kcal} / \mathrm{mol}$, which agree with the values of $4.31 \mathrm{kcal} / \mathrm{mol}$ from Nicovich and Ravishankara [51]. 


\subsection{Surface diffusion}

The impact of surface diffusion on the overall reaction rate is further investigated to reveal the full image of reaction dynamics of $\mathrm{H}$ atoms on PAHs. Figure 11a illustrates an example that the incident $\mathrm{H}$ atom undergoes an HB reaction after the third collision on the pocket sites of particle surface. A similar example for HD reaction is also included in Fig. 11a. The above two cases involve two reactions via surface diffusion, and we term this type of surface reactions as surface diffusion induced reaction. The number of sites that an incident $\mathrm{H}$ atom visits in each trajectory $\left(N_{\text {site }}\right)$ and its distribution for all five models are illustrated in Fig. 11b. Each row represents the distribution of $N_{\text {site }}$ for a particular case from 1500 to $2700 \mathrm{~K}$, and the median value of $N_{\text {site }}$ is marked by white dots. For PAH monomers, the median values of $N_{\text {site }}$ are 1 and 2-3 for surface $\mathrm{H}$ and $\mathrm{C}$ atoms, respectively. This is consistent with the finding in Fig. 8 as the incident $\mathrm{H}$ atom can access both $\mathrm{H}$ and $\mathrm{C}$ atoms at the same time in a single collision event. The $N_{\text {site }}$ of PAH monomers suggests that the incident $\mathrm{H}$ atom only visits the surface $\mathrm{H}$ atom once in one trajectory, but multiple times for surface $\mathrm{C}$ atoms. However, considering the overlap of surface $\mathrm{C}$ atoms, the incident $\mathrm{H}$ atom in fact collides on more than one $\mathrm{C}$ atoms at the same time, resulting in that the $N_{\text {site }}$ of $\mathrm{C}$ atom exceeds one. Therefore, the collisions between the incident $\mathrm{H}$ atoms and PAH monomers are governed by the specular scattering as expected. The distributions of $N_{\text {site }}$ for PAH monomers have a long tail toward tens of visited sites indicating that surface diffusion exists in the bimolecular collision to an extent. A larger PAH shows a longer tail, which is consistent with the ability of momentum accommodation [52]. For the quasi-surface models, both $N_{\text {site }}$ of C and $\mathrm{H}$ sites are higher than that of monomers on average, suggesting that diffusive scattering dominates the interactions between the incident $\mathrm{H}$ and surface atoms. 


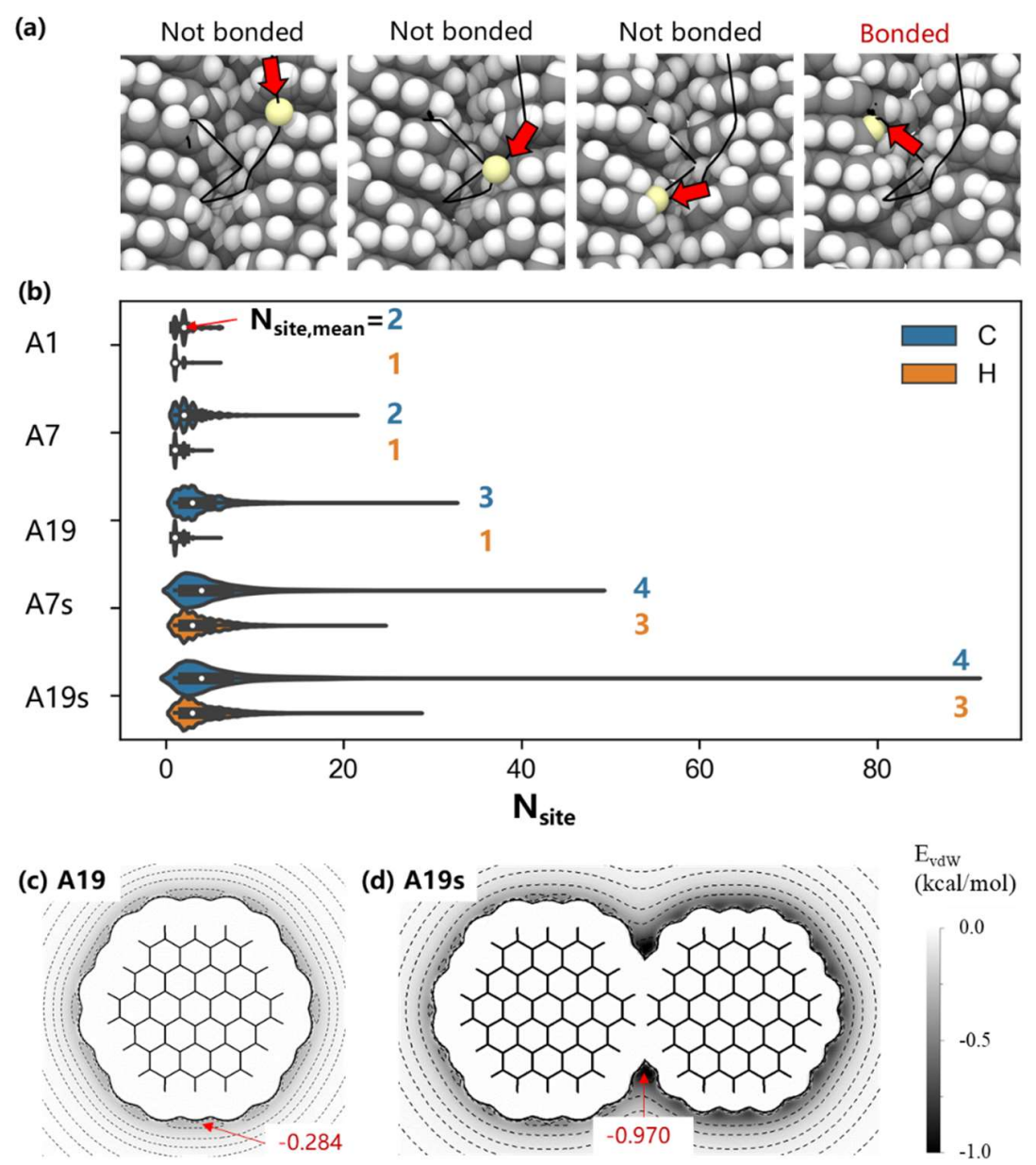

Figure 11 (a) Snapshots of trajectories for surface diffusion induced HB and HD reaction on A19s. (b) The number of $\mathrm{C}$ and $\mathrm{H}$ atoms visited by the incident $\mathrm{H}$ atom after the collisions on surface $\mathrm{H}$ atom. The shadow regions represent the detailed distribution. The white dots represent the median values, which are also highlighted on the right side of figure. The van der Waals potential energy surface of (c) A19 and (d) A19s. The cross section of the A19s is cut through the plane of the left A19 molecule. The minimum potential energies are highlighted by the red values.

We also learned that the $N_{\text {site }}$ is almost independent on the temperature (Fig. S9), however, this does not agree with the previous understanding of gas-nanoparticle scattering [49] as the kinetic energy of incident atoms impacts the scattering mode. This might be attributed to the good ability of energy accommodation in PAH molecules. To understand the discrepancy between the surface diffusion on PAH monomers and quasi-surfaces, the van der Waals potential energy surface of A19 and A19s were 
constructed following the method of Lu and Chen [53], and visualized using Multiwfn program [54]. An $\mathrm{H}$ atom was used as a probe to calculate the potential energy surface. A negative potential energy (i.e. $\left.E_{v d w}\right)$ indicates that the incident $\mathrm{H}$ atom is attracted by the neighboring atoms. Since the average incident kinetics energies of $\mathrm{H}$ atoms are in the range of $4-8 \mathrm{kcal} / \mathrm{mol}$ at $1500-2700 \mathrm{~K}$, which are comparable with the van der Waals potential on particle surface. It is expected that the incident $\mathrm{H}$ atoms exhibit weak attractions from surface atoms before collision. As shown in Fig. $11 \mathrm{c}$ and d, the $E_{v d w}$ values of A19s is larger than that of A19 (absolute value), suggesting that the attractions in the quasi-surface models are stronger. The spatial distribution of $E_{v d w}$ for A19 is uniform, while the pocket sites of quasi-surface models show a larger $E_{v d w}$ by $\sim 0.5 \mathrm{kcal} / \mathrm{mol}$ compared to the flat regions, and this supports the enhancement of collisions in the pocket sites (Fig. 8).

The representative collision dynamics of surface diffusion induced reactions is illustrated from the kinetic energy of the incident $\mathrm{H}$ atom on the surface of A19s (Fig. 12). In the first trajectory (Fig. 12a), the incident $\mathrm{H}$ atom undergoes a diffusive scattering. It gains $\sim 5 \mathrm{kcal} / \mathrm{mol}$ in the first collision at $\sim 260 \mathrm{ps,}$ then experiences multiple collision to gain and lose kinetics energy. Finally, it gains $\sim 9 \mathrm{kcal} / \mathrm{mol}$ from the last collision ( $\sim 1460 \mathrm{fs})$ to overcome the surface attraction (Fig. 11d) resulting in the desorption from the surface. Note that the kinetic energy exhibits a minor fluctuation during the surface diffusion. In Fig. 12b, the evolution of the kinetic energy of an $\mathrm{HB}$ reaction is presented, where the incident $\mathrm{H}$ atom reacts with surface $\mathrm{H}$ atom to release a $\mathrm{H}_{2}$ molecule. The incident $\mathrm{H}$ atom collides with the surface at $510 \mathrm{fs}$ and loses $\sim 4 \mathrm{kcal} / \mathrm{mol}$ in the first collision. It further undergoes surface diffusion on the surface of A19s, which involves 12 collisions in total. After the last collision at $\sim 2180 \mathrm{fs}$, the kinetic energy of the incident $\mathrm{H}$ atom reaches up to $20 \mathrm{kcal} / \mathrm{mol}$, which overcomes the energy barrier of $\mathrm{HB}$ reactions $(\sim 16.5 \mathrm{kcal} / \mathrm{mol})$ [7], and an $\mathrm{HB}$ reaction occurs. Similarly, the acceleration of $\mathrm{H}$ atom during the surface diffusion can lead to an $\mathrm{HD}$ reaction as well (Fig. 12c). 

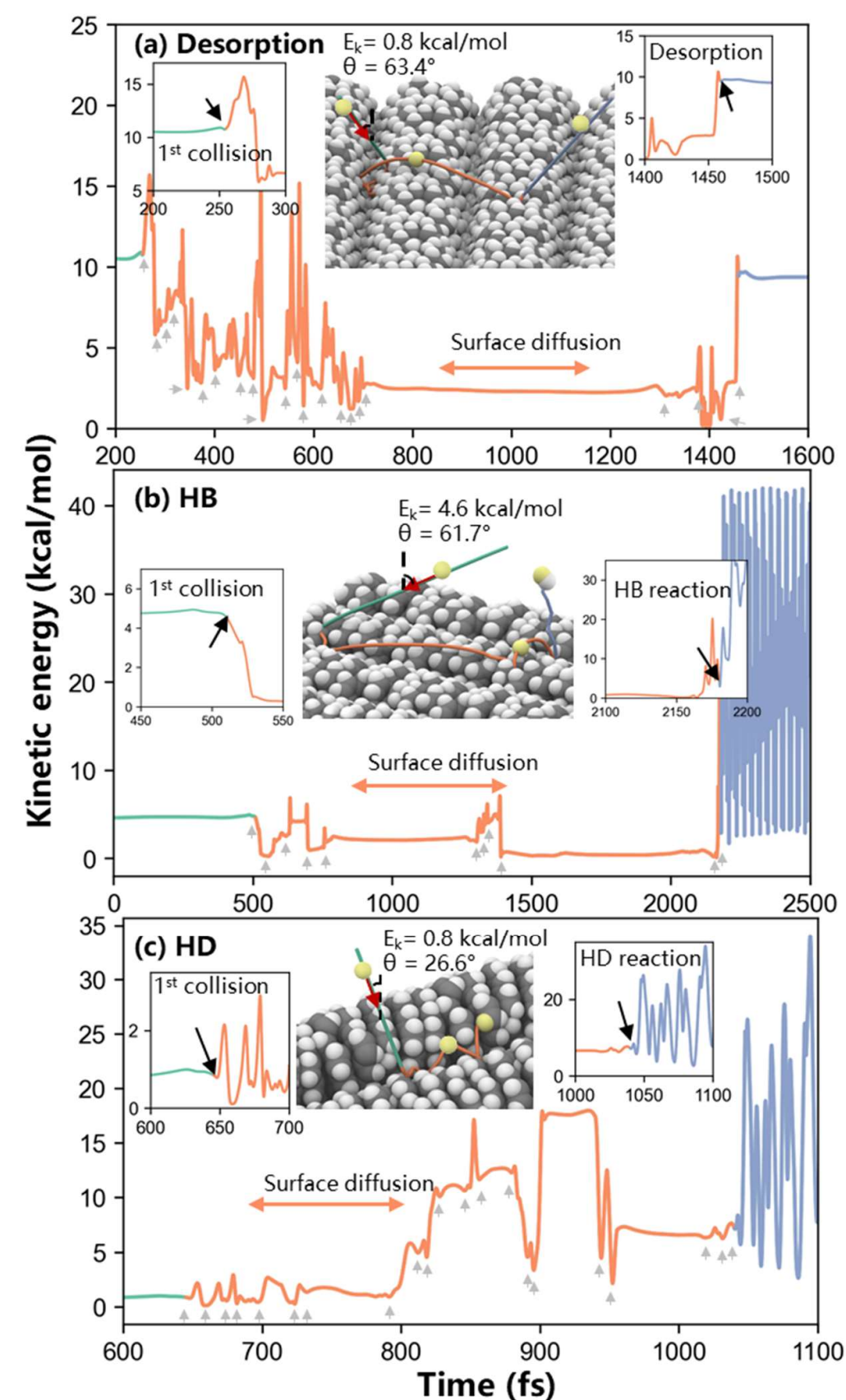

Figure 12. Kinetic energy profiles of an incident $\mathrm{H}$ atom in three representative trajectories involving surface diffusion: (a) desorption after non-reactive collisions, (b) HB reaction and (c) HD reaction. The grey arrows highlight the collisions between the incident $\mathrm{H}$ atom and surface atoms. Detailed animations of three representative trajectories are included in the Supplementary Materials. 
The nature of surface diffusion induced reactions lies in the energy transfer between the incident species and surface atoms. Once the incident species gains enough kinetic energy from surface diffusion, the potential surface reaction could be seen afterward. The previous knowledge neglects the potential reactions in the diffusive scattering [49], where the incident species loses its kinetic energy and no reaction proceeds. However, our findings here demonstrate the importance of surface diffusion induced reactions, and highlight the feasibility to gain kinetic energy from surface diffusion in the models of soot surface.

The probability of surface diffusion in all trajectories are discussed together with the proportions of surface diffusion induced HB and HD reactions (Fig. 13). We selected $N_{\text {site }, H}>2$ as a critical value to recognize the surface diffusion. The diffusive scattering only accounts for $0.91-1.44 \%$ of all collisions between the incident $\mathrm{H}$ and PAH monomers, while the probability of diffusive scattering is enhanced to 59.4-60.9\% for quasi-surface models. Both surface diffusion induced $\mathrm{HB}$ and $\mathrm{HD}$ reactions is insignificant $(\sim 4 \%)$ in the cases of PAH monomers as expected, and the reactions can be approximated as the product of a single collision. By contrast, $\sim 50 \%$ of both HB and HD reactions proceeds via multiple collisions between the incident $\mathrm{H}$ atom and surface atoms, that is, surface diffusion induced reactions. This proves that the surface diffusion induced reactions are critical in the treatment of surface reactions in soot formation. However, the surface diffusion induced reactions were not novel in surface science, and they were previously observed in the area of metal catalysis, for example, Langmuir-Hinshelwood (LH) mechanism. In LH mechanism, two reacting species are chemisorbed on the catalyst surface, and surface diffusion events drive the two species together before a reaction takes place [55]. Examples of the LH mechanism are seen in the systems of $\mathrm{H}+\mathrm{H}$-absorbed $\mathrm{Ni}(100)$ [56], $\mathrm{H}+\mathrm{Cl}$-adsorbed $\mathrm{Au}(111)$ [57] and $\mathrm{H}+\mathrm{H}$-absorbed graphite [58]. Nevertheless, the impact of surface diffusion on the surface reactions of soot should not be neglected, and further detailed analysis will help the development of surface models for soot. 


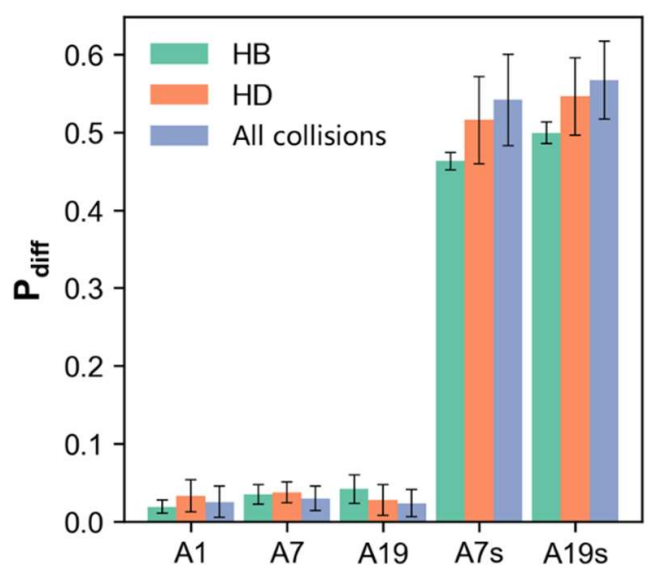

Figure 13. The contribution of surface diffusion $\left(P_{\text {diff }}\right)$ into the HB reactions, HD reactions and all collisions for cases of A1, A7, A19, A7s and A19s. The green, orange and blue represent the $P_{\text {diff }}$ in HB reactions, HD reactions and all collision events, respectively. The error bars are one standard deviation computed by the bootstrapping resampling method.

Soot particles have complex surface structures including cross-linking [59], heteroatoms [60] and curved PAHs [61], and the current model focuses on a series of simplified quasi-surface models that only involves $\mathrm{C}-\mathrm{H}$ sites. But we believe the surface diffusion induced reactions exist in other cases. When the surface evolves to carbon-rich configurations as soot become mature, the preferred reaction pathway might be switched from HB reactions to HD reactions. The HD reactions could super-hydrogenates the particle surface, which weaken the C-backbone and accelerate the fragmentation upon collisions or photoionization [62,63]. A further study on the fate of the product of HD reactions is critical to assess its impact on the surface reactions. Besides $\mathrm{H}$ atoms, other radical species, i.e. $\mathrm{OH}$ and $\mathrm{CH}_{3}$, also play an important role in the PAH chemistry. A competition between abstraction and addition reactions among these species is expected, and the rate of each reaction pathway depends on the local potential energy surface. 


\section{Conclusions}

The quasi-classical trajectories (QCT) method in combination with molecular geometric analysis are applied to explore the HB and HD reactions on PAH monomers and quasi-surface models from 1500 to $2700 \mathrm{~K}$. The molecular structure of monomers and quasi-surface models are investigated by solventexcluded surface (SES) area. For PAH monomers, it is found that the percentage of surface hydrogen atom $\left(\alpha_{H}\right)$ decreases with the PAH size, resulting in the preferred reaction pathway changing from $\mathrm{HB}$ to HD reactions. By contrast, the quasi-surface models exhibit weak dependence on the PAH size due to the similar $\alpha_{H}$. Furthermore, several reactive sites are highlighted to understand the effects of surface area, incident angle and van der Waals potential on the reaction dynamics. We observed the phenomenon of surface diffusion in the models of quasi-surface, where the incident $\mathrm{H}$ atom collides with several reactive sites. A portion of the surface diffusion results in the HB or HD reactions on particle surface due to the gain of kinetic energy from surface atoms. This type of unexpected reactions prevails in the models of the quasi-surface sites, resulting in one order of magnitude enhancement of reaction rates than monomer sites at the maximum.

Our study also highlights the misuse of small PAHs to represent the large PAHs or even soot particles for considering the underlying reaction kinetics, which might underestimate the magnitudes of surface reaction rates and misinterpret the reaction path in the worst cases. The key lies in the treatment of surface diffusion induced reactions. More future works are required to determine the intensity of surface diffusion and its impact on the reaction pathway among more complex surfaces (involving $\mathrm{C}$ rich, cross-linking, curved $\mathrm{PAH})$ and gas species $\left(\mathrm{OH}, \mathrm{CH}_{3}\right)$. However, the surface diffusion phenomena should always exist on particle surface, and becomes stronger as particle size increases. We anticipate that the surface reactions in the events of surface diffusion discovered here also applies to other gas-surface reactions, which is of critical significance for our understanding of chemical reactions in surface reactions. Also, we expect that 
our results can stimulate the development of experimental methods to explore such unexpected phenomenon from the basis of reaction kinetics in soot formation.

\section{Acknowledgements}

This work was supported by the National Natural Science Foundation of China (Grant No. 21961122007, 51806016, 11672314 and 51676016). DC also acknowledges the support by the Beijing Institute of Technology Research Fund Program for Young Scholars. 


\section{References}

[1] Niessner R. The many faces of soot: Characterization of soot nanoparticles produced by engines. Angew Chem - Int Ed 2014;53:12366-79. https://doi.org/10.1002/anie.201402812.

[2] Wang H. Formation of nascent soot and other condensed-phase materials in flames. Proc Combust Inst 2011;33:41-67. https://doi.org/10.1016/j.proci.2010.09.009.

[3] Alfè M, Apicella B, Barbella R, Rouzaud JN, Tregrossi A, Ciajolo A. Structure-property relationship in nanostructures of young and mature soot in premixed flames. Proc Combust Inst 2009;32 I:697704. https://doi.org/10.1016/j.proci.2008.06.193.

[4] Dobbins RA, Fletcher RA, Benner BA, Hoeft S. Polycyclic aromatic hydrocarbons in flames, in diesel fuels, and in diesel emissions. Combust Flame 2006;144:773-81. https://doi.org/10.1016/j.combustflame.2005.09.008.

[5] Singh J, Balthasar M, Kraft M, Wagner W. Stochastic modeling of soot particle size and age distributions in laminar premixed flames. Proc Combust Inst 2005;30:1457-65. https://doi.org/10.1016/j.proci.2004.08.120.

[6] Sander M, Patterson RIA, Braumann A, Raj A, Kraft M. Developing the PAH-PP soot particle model using process informatics and uncertainty propagation. Proc Combust Inst 2011;33:675-83. https://doi.org/10.1016/j.proci.2010.06.156.

[7] Hou D, You X. Reaction kinetics of hydrogen abstraction from polycyclic aromatic hydrocarbons by H atoms. Phys Chem Chem Phys 2017;19:30772-80. https://doi.org/10.1039/C7CP04964A.

[8] Liu P, Li Z, Bennett A, Lin H, Sarathy SM, Roberts WL. The site effect on PAHs formation in HACA-based mass growth process. Combust Flame 2019;199:54-68. https://doi.org/10.1016/j.combustflame.2018.10.010.

[9] Frenklach M. Reaction mechanism of soot formation in flames. Phys Chem Chem Phys 2002;4:2028-37. https://doi.org/10.1039/b110045a.

[10] Frenklach M. On surface growth mechanism of soot particles. Symp Int Combust 1996;26:2285-93. https://doi.org/10.1016/S0082-0784(96)80056-7.

[11] Mao Q, van Duin ACT, Luo KH. Formation of incipient soot particles from polycyclic aromatic hydrocarbons: A ReaxFF molecular dynamics study. Carbon 2017;121:380-8. https://doi.org/10.1016/j.carbon.2017.06.009.

[12] Totton TS, Misquitta AJ, Kraft M. A quantitative study of the clustering of polycyclic aromatic hydrocarbons at high temperatures. Phys Chem Chem Phys 2012;14:4081-94. https://doi.org/10.1039/c2cp23008a.

[13] Chen D, Totton TS, Akroyd JWJ, Mosbach S, Kraft M. Size-dependent melting of polycyclic aromatic hydrocarbon nano-clusters: A molecular dynamics study. Carbon 2014;67:79-91. https://doi.org/10.1016/j.carbon.2013.09.058.

[14] Chen D, Luo KH. Reactive sites on the surface of polycyclic aromatic hydrocarbon clusters: A numerical study. Combust Flame 2020;211:362-73. https://doi.org/10.1016/j.combustflame.2019.09.034.

[15] Chen D, Akroyd J, Mosbach S, Kraft M. Surface reactivity of polycyclic aromatic hydrocarbon clusters. Proc Combust Inst 2015;35:1811-8. https://doi.org/10.1016/j.proci.2014.06.140.

[16] Akter MH. A quasiclassical trajectory study of $\mathrm{H}_{2}+\mathrm{H}_{2}$ energy transfer: a survey of applicability of detailed balance. Masters. University of Northern British Columbia, 2016. https://doi.org/10.24124/2016/1215. 
[17] Zhu Y, Tian L, Song H, Yang M. Kinetic and dynamic studies of the $\mathrm{H}_{3}{ }^{+}+\mathrm{CO} \rightarrow \mathrm{H}_{2}+\mathrm{HCO}^{+}$ $/ \mathrm{HOC}^{+}$reaction on a high-level ab initio potential energy surface. J Chem Phys 2019;151:054311. https://doi.org/10.1063/1.5110934.

[18] Yu H-G. Product Branching Ratios of the Reaction of $\mathrm{CO}$ with $\mathrm{H}^{+}{ }_{3}$ and $\mathrm{H}_{2} \mathrm{D}^{+}$. Astrophys $\mathrm{J}$ 2009;706:L52-5. https://doi.org/doi:10.1088/0004-637X/706/1/L52.

[19] Zhao J, Xu Y, Yue D, Meng Q. Quasi-classical trajectory study of the reaction $\mathrm{H}+\mathrm{FO} \rightarrow \mathrm{OH}+\mathrm{F}$. Chem Phys Lett 2009:3. https://doi.org/10.1016/j.cplett.2009.02.007.

[20] Fu Y-L, Lu X, Han Y-C, Fu B, Zhang DH, Bowman JM. Collision-induced and complex-mediated roaming dynamics in the $\mathrm{H}+\mathrm{C}_{2} \mathrm{H}_{4} \rightarrow \mathrm{H}_{2}+\mathrm{C}_{2} \mathrm{H}_{3}$ reaction. Chem Sci 2020;11:2148-54. https://doi.org/10.1039/C9SC05951B.

[21] Happold J, Grotheer H-H, Aigner M. Soot precursors consisting of stacked pericondensed PAHs. KIT Scientific Publishing, Karlsruhe, Germany; 2009.

[22] Commodo M, Kaiser K, De Falco G, Minutolo P, Schulz F, D'Anna A, et al. On the early stages of soot formation: Molecular structure elucidation by high-resolution atomic force microscopy. Combust Flame 2019;205:154-64. https://doi.org/10.1016/j.combustflame.2019.03.042.

[23] Yu H-G, Muckerman JT. Ab Initio and Direct Dynamics Studies of the Reaction of Singlet Methylene with Acetylene and the Lifetime of the Cyclopropene Complex. J Phys Chem A 2005;109:1890-6. https://doi.org/10.1021/jp045049w.

[24] Kholghy MR, Veshkini A, Thomson MJ. The core-shell internal nanostructure of soot - A criterion to model soot maturity. Carbon 2016;100:508-36. https://doi.org/10.1016/j.carbon.2016.01.022.

[25] Hurt RH, Crawford GP, Shim H-S. Equilibrium nanostructure of primary soot particles. Proc Combust Inst 2000;28:2539-46. https://doi.org/10.1016/S0082-0784(00)80670-0.

[26] Jiang Y, Deng S, Hong S, Zhao J, Huang S, Wu C-C, et al. Energetic Performance of Optically Activated Aluminum/Graphene Oxide Composites. ACS Nano 2018;12:11366-75. https://doi.org/10.1021/acsnano.8b06217.

[27] Wang S, Zhou G, Ma Y, Gao L, Song R, Jiang G, et al. Molecular dynamics investigation on the adsorption behaviors of $\mathrm{H}_{2} \mathrm{O}, \mathrm{CO}_{2}, \mathrm{CH}_{4}$ and $\mathrm{N}_{2}$ gases on calcite $\left(11^{-} 0\right)$ surface. Appl Surf Sci 2016;385:616-21. https://doi.org/10.1016/j.apsusc.2016.05.026.

[28] Sirignano M, Collina A, Commodo M, Minutolo P, D'Anna A. Detection of aromatic hydrocarbons and incipient particles in an opposed-flow flame of ethylene by spectral and time-resolved laser induced emission spectroscopy. Combust Flame 2012;159:1663-9. https://doi.org/10.1016/j.combustflame.2011.11.005.

[29] Plimpton S. Fast Parallel Algorithms for Short - Range Molecular Dynamics. J Comput Phys 1995;117:1-19. https://doi.org/10.1006/jcph.1995.1039.

[30] Mao Q, Luo KH. Trace metal assisted polycyclic aromatic hydrocarbons fragmentation, growth and soot nucleation. Proc Combust Inst 2019;37:1023-30. https://doi.org/10.1016/j.proci.2018.06.106.

[31] Mao Q, Ren Y, Luo KH, van Duin ACT. Dynamics and kinetics of reversible homo-molecular dimerization of polycyclic aromatic hydrocarbons. J Chem Phys 2017;147:244305. https://doi.org/10.1063/1.5000534.

[32] Sanner MF, Olson AJ, Spehner J-C. Reduced surface: an efficient way to compute molecular surfaces. Biopolymers 1996;38:305-320. https://doi.org/10.1002/(SICI)1097-0282(199603)38:3<305::AIDBIP4>3.0.CO;2-Y.

[33] Bondi A. Van der Waals volumes and radii. J Phys Chem 1964;68:441-451. https://doi.org/10.1021/j100785a001. 
[34] Johnson R. Computational Chemistry Comparison and Benchmark Database, NIST Standard Reference Database 101 2002. https://doi.org/10.18434/T47C7Z.

[35] Nevitt J, Hancock GR. Performance of bootstrapping approaches to model test statistics and parameter standard error estimation in structural equation modeling. Struct Equ Model 2001;8:353377. https://doi.org/10.1207/S15328007SEM0803 2.

[36] Hou D, Zong D, Lindberg CS, Kraft M, You X. On the coagulation efficiency of carbonaceous nanoparticles. J Aerosol Sci 2020;140:105478. https://doi.org/10.1016/j.jaerosci.2019.105478.

[37] Foley N, Cazaux S, Egorov D, Boschman LMPV, Hoekstra R, Schlatholter T. Molecular hydrogen formation on interstellar PAHs through Eley-Rideal abstraction reactions. Mon Not R Astron Soc 2018;479:649-56. https://doi.org/10.1093/mnras/sty1528.

[38] Park J, Dyakov IV, Lin MC. FTIR and Mass-Spectrometric Measurements of the Rate Constant for the $\mathrm{C}_{6} \mathrm{H}_{5}+\mathrm{H}_{2}$ Reaction. J Phys Chem A 1997;101:8839-43. https://doi.org/10.1021/jp972162w.

[39] Jiang H, Kammler M, Ding F, Dorenkamp Y, Manby FR, Wodtke AlecM, et al. Imaging covalent bond formation by $\mathrm{H}$ atom scattering from graphene. Science 2019;364:379-82. https://doi.org/10.1126/science.aaw6378.

[40] Seminara GN, Peludhero IF, Dong W, Martínez AE, Busnengo HF. Molecular Dynamics Study of Molecular and Dissociative Adsorption Using System-Specific Force Fields Based on Ab Initio Calculations: $\quad \mathrm{CO} / \mathrm{Cu}$ (110) and $\mathrm{CH}{ }_{4} / \mathrm{Pt}$ (110). Top Catal 2019;62:1044-52. https://doi.org/10.1007/s11244-019-01196-9.

[41] Valentini P, Schwartzentruber TE, Cozmuta I. Molecular dynamics simulation of O2 sticking on $\mathrm{Pt}_{(111)}$ using the $a b$ initio based ReaxFF reactive force field. J Chem Phys 2010;133:084703. https://doi.org/10.1063/1.3469810.

[42] Dahneke BE. Slip correction factors for nonspherical bodies-II free molecule flow. J Aerosol Sci 1973;4:147-161. https://doi.org/10.1016/0021-8502(73)90066-9.

[43] Epstein PS. On the resistance experienced by spheres in their motion through gases. Phys Rev 1924;23:710. https://doi.org/10.1103/PhysRev.23.710.

[44] Millikan RA. Coefficients of slip in gases and the law of reflection of molecules from the surfaces of solids and liquids. Phys Rev 1923;21:217. https://doi.org/ttps://doi.org/10.1103/PhysRev.21.217.

[45] Chen D, Wang K, Wang H. Violation of collision limit in recently published reaction models. Combust Flame 2017;186:208-10. https://doi.org/10.1016/j.combustflame.2017.08.005.

[46] Golden DM, Frenklach M, Moriarty NW, Eiteneer B, Goldenberg M, Bowman CT, et al. GRI-MECH 3.0 2000. http://www.me.berkeley.edu/gri mech/.

[47] Wang H, You X, Ameya VJ, Scott GD. USC Mech Version II. High-Temperature Combustion Reaction Model of $\mathrm{H}_{2} / \mathrm{CO} / \mathrm{C} 1-\mathrm{C} 4$ Compounds. 2007. http: //ignis.usc.edu/USC_Mech_II.htm.

[48] Metcalfe WK, Burke SM, Ahmed SS, Curran HJ. A Hierarchical and Comparative Kinetic Modeling Study of C1-C2 Hydrocarbon and Oxygenated Fuels. Int J Chem Kinet 2013;45:638-75. https://doi.org/10.1002/kin.20802.

[49] Li Z, Wang H. Gas-Nanoparticle Scattering: A Molecular View of Momentum Accommodation Function. Phys Rev Lett 2005;95:014502. https://doi.org/10.1103/PhysRevLett.95.014502.

[50] Raj A, Celnik M, Shirley R, Sander M, Patterson R, West R, et al. A statistical approach to develop a detailed soot growth model using PAH characteristics. Combust Flame 2009;156:896-913. https://doi.org/10.1016/j.combustflame.2009.01.005.

[51] Nicovich JM, Ravishankara AR. Reaction of hydrogen atom with benzene. Kinetics and mechanism. J Phys Chem 1984;88:2534-41. https://doi.org/10.1021/j150656a021. 
[52] Wang H, Wen K, You X, Mao Q, Luo KH, Pilling MJ, et al. Energy transfer in intermolecular collisions of polycyclic aromatic hydrocarbons with bath gases $\mathrm{He}$ and Ar. J Chem Phys 2019;151:044301. https://doi.org/10.1063/1.5094104.

[53] Lu T, Chen Q. Van der Waals potential: an important complement to molecular electrostatic potential in studying intermolecular interactions. ChemRxiv 2020:1-16. https://doi.org/10.26434/chemrxiv.12148572.v2.

[54] Lu T, Chen F. Multiwfn: A multifunctional wavefunction analyzer. J Comput Chem 2012;33:58092. https://doi.org/10.1002/jcc.22885.

[55] Rettner CT, Auerbach DJ. Distinguishing the Direct and Indirect Products of a Gas-Surface Reaction. Science 1994;263:365-7. https://doi.org/10.1126/science.263.5145.365.

[56] Guvenc ZB, Sha X, Jackson B. The Effects of Lattice Motion on Eley-Rideal and Hot Atom Reactions: Quasiclassical Studies of Hydrogen Recombination on Ni(100) n.d.:7.

[57] Quattrucci JG, Jackson B, Lemoine D. Eley-Rideal reactions of $\mathrm{H}$ atoms with $\mathrm{Cl}$ adsorbed on $\mathrm{Au}(111)$ : Quantum and quasiclassical studies. J Chem Phys 2003;118:2357-66. https://doi.org/10.1063/1.1533735.

[58] Martinazzo R, Tantardini GF. Quantum study of Eley-Rideal reaction and collision induced desorption of hydrogen atoms on a graphite surface. I. H-chemisorbed case. J Chem Phys n.d.:15.

[59] Martin JW, Hou D, Menon A, Pascazio L, Akroyd J, You X, et al. Reactivity of Polycyclic Aromatic Hydrocarbon Soot Precursors: Implications of Localized $\pi$-Radicals on Rim-Based Pentagonal Rings. J Phys Chem C 2019;123:26673-82. https://doi.org/10.1021/acs.jpcc.9b07558.

[60] Johansson KO, Dillstrom T, Monti M, El Gabaly F, Campbell MF, Schrader PE, et al. Formation and emission of large furans and oxygenated hydrocarbons from flames. Proc Natl Acad Sci 2016;113:8374-9. https://doi.org/10.1073/pnas.1604772113.

[61] Martin JW, Bowal K, Menon A, Slavchov RI, Akroyd J, Mosbach S, et al. Polar curved polycyclic aromatic hydrocarbons in soot formation. Proc Combust Inst 2019;37:1117-23. https://doi.org/10.1016/j.proci.2018.05.046.

[62] Gatchell M, Stockett MH, de Ruette N, Chen T, Giacomozzi L, Nascimento RF, et al. Failure of hydrogenation in protecting polycyclic aromatic hydrocarbons from fragmentation. Phys Rev A 2015;92:050702. https://doi.org/10.1103/PhysRevA.92.050702.

[63] Wolf M, Giacomozzi L, Gatchell M, de Ruette N, Stockett MH, Schmidt HT, et al. Hydrogenated pyrene: Statistical single-carbon loss below the knockout threshold. Eur Phys J D 2016;70:85. https://doi.org/10.1140/epjd/e2016-60735-3. 